Research Article

\title{
Bodenverdichtung im Dauergrünland und ihre Auswirkung auf die Grünlandvegetation
}

\section{Soil compaction in permanent grassland and its effect on grassland vegetation}

\author{
Andreas Bohner ${ }^{1 *}$, Philipp Gehmacher ${ }^{2}$, Gernot Bodner ${ }^{3}$, Peter Strauss ${ }^{4}$
}

\footnotetext{
${ }^{1}$ Höhere Bundeslehr- und Forschungsanstalt Raumberg-Gumpenstein, Raumberg 38, 8952 Irdning-Donnersbachtal, Österreich

${ }^{2}$ Voglhütte 1, 5162 Obertrum am See, Österreich

${ }^{3}$ Universität für Bodenkultur, Abteilung Pflanzenbau, Konrad-Lorenz-Straße 24, 3430 Tulln, Österreich

${ }^{4}$ Bundesamt für Wasserwirtschaft, Institut für Kulturtechnik und Bodenwasserhaushalt, Pollnbergstraße 1, 3252 Petzenkirchen, Österreich

* Korrespondierender Autor: andreas.bohner@raumberg-gumpenstein.at
}

Received: 20 July 2017, received in revised form: 14 September 2017, accepted: 14 September 2017

\section{Zusammenfassung}

Die Bodenverdichtung ist ein aktuelles Problem der intensiven Grünlandwirtschaft. Daher wurden in einem repräsentativen österreichischen Grünlandgebiet an 22 unterschiedlichen Standorten die Lagerungsdichte (LD), der Eindringwiderstand (EW) und das Porenvolumen von typischen Dauergrünlandböden (Braunerde, Pseudogley, Gley) bis zu einer Bodentiefe von $30 \mathrm{~cm}$ bestimmt, um die bodenverdichtende Wirkung einer intensiven Grünlandbewirtschaftung beurteilen zu können. Außerdem wurde geprüft, ob zwischen den bodenphysikalischen Parametern und dem Auftreten von Bodenverdichtungszeigern im Pflanzenbestand ein Zusammenhang existiert. Der Verdichtungszustand von Dauergrünlandböden hängt von den Bodeneigenschaften (Humusgehalt, Bodenart), der Bewirtschaftungsform und der Nutzungsintensität ab. Die Verdichtungsempfindlichkeit sinkt mit steigendem Humus- und Tongehalt im Boden. Bei hoher Nutzungsintensität sind die Dauergrünlandböden mäßig überverdichtet. Die stärkste Bodenverdichtung entsteht infolge intensiver Beweidung mit Rindern. Durch Viehtritt wird der Boden insbesondere bis in $15 \mathrm{~cm}$ Tiefe verdichtet. Häufiges Befahren mit Grünlandmaschinen bewirkt eine Verdichtung zumindest bis $25 \mathrm{~cm}$ Bodentiefe. Die Mähweiden nehmen hinsichtlich Bodenverdichtung eine Mittelstellung zwischen Wiesen und Weiden ein. Die Gefahr einer ertragsmindernden Schadverdichtung steigt in Lehmböden beträchtlich, wenn innerhalb der oberen Bodenschicht $(0-20 \mathrm{~cm})$ eine Verdichtungszone mit einer LD über 1,40 g/ $\mathrm{cm}^{3}$ oder einem EW größer als 2,00 MPa auftritt. Die Bodenverdichtung wird im Dauergrünland durch Zeigerpflanzen zuverlässig indiziert. Ranunculus repens ist ein besonders sensitiver Bioindikator für Bodenverdichtung.

Schlagworte: Grünlandböden, Lagerungsdichte, Eindringwiderstand, Porenvolumen, Bodenverdichtungszeiger

\section{Summary}

Soil compaction caused by intensive grassland management is a worldwide problem. Therefore, in typical permanent grassland soils (Cambisol, Planosol, Gleysol) bulk density (BD), penetration resistance (PR) and pore volume to a depth of $30 \mathrm{~cm}$ at 22 different sites in a representative Austrian grassland region were determined. Moreover, the relationships between these soil properties and the incidence of indicator species for soil compaction were evaluated. Our results show that the compaction status depends on soil properties and type and intensity of grassland management. The susceptibility to compaction decreases with increasing humus and clay content in the soil. At high intensity of use, permanent grassland soils can be classified as moderately compacted. Intensive cattle treading has the greatest compacting effect, leading to compaction mainly in the uppermost $15 \mathrm{~cm}$ of the soil. Tractor traffic causes soil compaction to a depth of at least $25 \mathrm{~cm}$. Mowing pastures are intermediate between meadows and pastures concerning extent and depth of soil compaction. A loamy grassland soil is harmfully compacted if a compacted layer $\left(\mathrm{BD}>1,40 \mathrm{~g} / \mathrm{cm}^{3} \mathrm{or} P R>\right.$ 2,00 MPa) within the uppermost $20 \mathrm{~cm}$ of the soil occurs. Indicator species such as Ranunculus repens are suitable for identifying soil compaction.

Keywords: grassland soils, bulk density, penetration resistance, pore volume, indicator species for soil compaction 


\section{Einleitung}

Das Dauergrünland wird in Österreich als Wiese, Mähweide oder Weide genutzt (BMLFUW, 2016). Es findet weder ein Umbruch des Bodens noch eine ackerbauliche Nutzung innerhalb von zehn Jahren statt (Peeters et al., 2014). In den vergangenen Jahrzehnten hat vor allem in Regionen mit hohem Ertragspotenzial und günstigen Geländeverhältnissen eine Intensivierung der Grünlandbewirtschaftung stattgefunden (Dierschke und Briemle, 2002). Diese wird durch den Klimawandel und den Strukturwandel in der österreichischen Landwirtschaft auf vielen Standorten vermutlich weitergehen. Die mögliche Intensivierung bewirkt eine stärkere mechanische Belastung von Vegetation und Boden, weil die Grünlandflächen häufiger mit landwirtschaftlichen Maschinen befahren und/oder intensiver beweidet werden. Außerdem haben sowohl das Körpergewicht der Rinder, als auch die Größe und das Gewicht der Grünlandmaschinen, deutlich zugenommen (Spiekers und Potthast, 2003; Moitzi und Boxberger, 2007), weshalb die Bodenbelastung durch Viehtritt und Befahren beträchtlich angestiegen ist. Dies kann zu einer Veränderung der Struktur im Oberboden (Plattenbildung) und zu einer Verdichtung des Grünlandbodens (Sackungsverdichtung) im Ober- und Unterboden führen. Für die Oberbodenverdichtung ist der Kontaktflächendruck und für die Unterbodenverdichtung die Last (Gewicht von Maschinen oder Tieren) ausschlaggebend (Sommer, 1985). Rinder verursachen unter der Annahme von $700 \mathrm{~kg}$ Lebendgewicht einen mittleren Kontaktflächendruck von etwa 10 bar. Ein Allradtraktor mit einem Eigengewicht von $2240 \mathrm{~kg}$ hingegen erzeugt einen mittleren Kontaktflächendruck von 0,6 bar (Moitzi und Boxberger, 2007). Die Auswirkungen einer Bodenverdichtung auf den Pflanzenstandort sind vielfältig: Zunahme der Lagerungsdichte (LD) im Boden, Abnahme des Gesamt-Porenvolumens (PV), Veränderung der Porengrößenverteilung (bevorzugter Verlust an Grobporen), Unterbrechung der Porenkontinuität und Absenkung der Bodenoberfläche (Hartge und Horn, 2014). Im Grünland ist eine Absenkung von bis zu $20 \mathrm{~cm}$ möglich (ersichtlich an aufgewölbten Flächen unter Weidezäunen). Durch Bodenverdichtung werden nahezu alle physikalischen, chemischen und biologischen Bodeneigenschaften und -prozesse beeinflusst (Hakansson und Medveder, 1995). Das Luftvolumen im Boden wird vermindert und die Bodendurchlüftung (Gasdiffusion) verschlechtert. Überverdichtete Böden haben deshalb niedrigere Sauerstoff- und höhere Kohlendioxid-Konzentrationen in der Bodenluft als normalverdich- tete Böden (Hansen und Bakken, 1993). Durch Reduktion der Grobporen wird der Eindringwiderstand (EW) für Pflanzenwurzeln erhöht. Der EW des Bodens ist von der LD und vom aktuellen Bodenwassergehalt abhängig. Er steigt mit zunehmender LD und nimmt mit zunehmendem Bodenwassergehalt $\mathrm{ab}$, insbesondere in verdichteten Böden (Iijima und Kato, 2007; Herbin et al., 2011). Der EW wird stärker von der LD als vom aktuellen Bodenwassergehalt beeinflusst; der Einfluss des Bodenwassergehaltes nimmt allerdings mit steigendem Tongehalt im Boden zu (Hernanz et al., 2000). Ein hoher EW kann, ebenso wie mangelnde Durchlüftung, die Durchwurzelbarkeit des Bodens einschränken (Mohr, 1980; Evans et al., 2012; Pulido et al., 2017). Die Wasser- und Nährstoffvorräte im Boden werden dadurch schlechter ausgenutzt (Kozlowski, 1999). In überverdichteten Böden reduzieren die Pflanzenwurzeln ihr Längenwachstum, die Ausbildung von Seitenwurzeln wird hingegen verstärkt (Bengough et al., 2006). Eine starke Konzentration der Wurzelmasse auf die oberste Bodenschicht und eine schlechte Durchwurzelung des Unterbodens sind charakteristisch für überverdichtete Dauergrünlandböden (Bohner et al., 2016). Außerdem wird durch Bodenverdichtung die hydraulische Leitfähigkeit im Boden vermindert (Voorhees et al., 1986), weshalb Staunässebildung und Reduktionsprozesse (Herbauts et al., 1996) gefördert werden. Überverdichtete Grünlandböden sind daher vor allem in niederschlagreichen Regionen häufig krumen-pseudovergleyt. Roströhren zeigen die Staunässe im Oberboden an. Staunässe bewirkt einen zeitweiligen Sauerstoffmangel im Hauptwurzelraum, eine langsamere Bodenerwärmung und eine Veränderung in der Artenzusammensetzung der Grünlandvegetation (Bohner et al., 2006). Die Bewirtschaftbarkeit der Grünlandflächen wird erschwert, weil die Tragfähigkeit des Bodens und die Trittfestigkeit der Grasnarbe bodenfeuchtebedingt zeitweilig abnehmen (verringerte Zahl an Weidetagen, erschwerte Bedingungen bei der Futterwerbung und Düngung, erhöhte Gefahr der Ansiedlung und Ausbreitung unerwünschter Pflanzenarten durch Narbenschäden). In Hanglagen erhöht sich bei Starkregen mit zunehmender Bodenverdichtung aufgrund einer verringerten Wasserinfiltration (Chyba et al., 2014) der Oberflächenabfluss. Die Grundwasserneubildung wird dadurch vermindert, die Hochwassergefahr steigt, die Abschwemmung von Nährstoffen und Eutrophierung von Oberflächengewässern werden gefördert (Kurz et al., 2006). Durch Bodenverdichtung wird die Wärmeleitfähigkeit im Boden erhöht; geringere Temperaturschwankungen im Oberboden und in der bodennahen Luftschicht sind die 
Folge (Hartge und Horn, 2014). Durch Bodenverdichtung werden die Kohlenstoff- und Stickstoffmineralisation im Boden (Breland und Hansen, 1996; Nawaz et al., 2013) sowie die Netto-Nitrifikationsrate und biologische StickstoffFixierung (Hakansson und Medveder, 1995) vermindert, während Denitrifikationsprozesse im Boden (Nawaz et al., 2013) und Lachgasemissionen (Sitaula et al., 2000) erhöht werden. Die Bodenverdichtung begünstigt die Emission von Treibhausgasen $\left(\mathrm{CO}_{2}, \mathrm{~N}_{2} \mathrm{O}, \mathrm{CH}_{4}\right)$ und beeinflusst somit auch die Atmosphäre (Nawaz et al., 2013). Sie engt den Lebensraum von Bodenorganismen durch Verkleinerung des besiedelbaren Porenraumes ein und beeinflusst Abundanz, Diversität und Biomasse der Bodenorganismen (Larink et al., 1994). Nachteilige Folgen einer Bodenverdichtung können Ertragsrückgänge und eine verminderte Stickstoffaufnahme durch die Grünlandvegetation sein (Douglas und Crawford, 1993). Die Bodenverdichtung kann somit im Dauergrünland zum wachstumsbegrenzenden Faktor werden (Stahl et al., 2009). Eine natürliche Regeneration erfolgt sehr langsam und ist meist auf die oberste Bodenschicht $(0-15 \mathrm{~cm})$ beschränkt (Drewry, 2006). Auch aus naturschutzfachlicher Sicht ist eine Bodenverdichtung meistens negativ, vereinzelt allerdings auch positiv zu bewerten. Spiranthes spiralis (Herbst-Drehwurz) beispielsweise ist eine seltene und stark gefährdete Orchideenart. Sie profitiert von einer mäßigen Oberbodenverdichtung, weil dadurch Nagetiere vom Verzehr der Wurzelknollen abgehalten werden (Kriedner, 1989).

Da Bodenverdichtung eine wesentliche Ursache der physikalischen Bodendegradation ist (Drewry et al., 2008), müssen verdichtungsgefährdende Bewirtschaftungsmaßnahmen erkannt und soweit wie möglich vermieden werden. Zum Schutz des Bodens und der Umwelt sollten standortspezifische Intensivierungsgrenzen für die Grünlandbewirtschaftung festgelegt werden. Dazu sind Indikatoren und Schwellenwerte notwendig. Zeigerpflanzen sind als Indikatoren für die Nutzungsintensität und den Verdichtungsgrad von Dauergrünlandböden besonders geeignet, weil sie auf Veränderungen der Umweltfaktoren schneller und sensibler als viele Bodenparameter reagieren, weil sie flächendeckende Informationen über den Standort liefern und weil sie im Gelände einfach und rasch zu bestimmen sind. Unklar ist allerdings, ob das Vorkommen von Bodenverdichtungszeigern im Pflanzenbestand kausal mit der Bodenverdichtung oder mit der Nutzungsintensität zusammenhängt.

Die Bodenverdichtung wurde in Österreich vor allem in Ackerböden untersucht (Murer et al., 2006). In Dauergrünlandböden hingegen wurden bisher nur sehr wenige
Studien durchgeführt (Bohner et al., 2006; 2016). Wegen aktueller Bodenverdichtungsprobleme auf zahlreichen Grünlandflächen sind Untersuchungen zur Bodenverdichtung im österreichischen Dauergrünland dringend notwendig. Für die Feststellung einer Bodenverdichtung gibt es eine Vielzahl an bodenphysikalischen Parametern (Hartge und Horn, 2009). Häufig verwendete Messgrößen sind die LD und der EW des Bodens (Herbin et al., 2011; Newell-Price et al., 2013). Die Datenbasis für die LD und den EW ist in österreichischen Dauergrünlandböden schmal, da im Rahmen der routinemäßigen Bodenuntersuchung diese Parameter nicht gemessen werden. Bisher nicht befriedigend beantwortet ist die Frage, wie weit eine Bodenverdichtung im Dauergrünland tolerierbar ist und ab welchem Schwellenwert eine Schadverdichtung auftritt. Nach Stahl et al. (2009) sind im Grünland eine gesättigte Wasserleitfähigkeit unterhalb von $8 \mathrm{~cm} / \mathrm{d}$ oder eine Luftkapazität von unter 8 Vol.-\% wichtige Kriterien für das Vorliegen einer Bodenschadverdichtung. Nach Lebert et al. (2004) sind Böden schadverdichtet, wenn sie eine Luftkapazität unter 5 Vol.-\% und eine gesättigte Wasserleitfähigkeit unter $10 \mathrm{~cm} / \mathrm{d}$ aufweisen. Für die häufiger verwendeten Parameter LD und EW fehlen entsprechende Schwellenwerte. Sie werden im Dauergrünland zur Vermeidung von verdichtungsbedingten Ertragsminderungen dringend benötigt.

Mit dieser Arbeit werden folgende Ziele verfolgt:

1. Bereitstellung von Daten über die LD, den EW und das PV von typischen mineralischen Dauergrünlandböden bis in $30 \mathrm{~cm}$ Bodentiefe,

2. Evaluierung der Eignung von Zeigerpflanzen für die Feststellung einer Bodenverdichtung im Dauergrünland und

3. Ermittlung von Schwellenwerten für die Bodenschadverdichtung im Dauergrünland.

\section{Material und Methoden}

\subsection{Untersuchungsgebiet}

Das Untersuchungsgebiet befindet sich im nördlichen Salzburger Flachgau in den Gemeinden Obertrum am See $\left(47^{\circ} 56^{\prime} \mathrm{N}, 13^{\circ} 04^{\prime} \mathrm{E}\right)$, Anthering $\left(47^{\circ} 52^{\prime} \mathrm{N}, 13^{\circ} 00^{\prime} \mathrm{E}\right)$ und Seekirchen am Wallersee $\left(47^{\circ} 53^{\prime} \mathrm{N}, 13^{\circ} 08^{\prime} \mathrm{E}\right)$. Die Landschaft repräsentiert ein flachwelliges Hügelland mit Seehöhen zwischen 400 und $850 \mathrm{~m}$. An der Wetterstation Mattsee (504 m Seehöhe) betragen im langjährigen Mittel (1971-2000) die Jahresmittel-Temperatur 8,5 ${ }^{\circ} \mathrm{C}$, 
die Juli-Temperatur $18,1^{\circ} \mathrm{C}$ und die Jänner-Temperatur $-1,1^{\circ} \mathrm{C}$. Der Jahres-Niederschlag macht im Durchschnitt $1333 \mathrm{~mm}$ aus. Die mittlere Monatssumme des Niederschlags variiert zwischen 70 (Februar) und $160 \mathrm{~mm}$ (Juli). Die Zahl der Frosttage beträgt im langjährigen Mittel 97 im Jahr (ZAMG, 2002).

Das Untersuchungsgebiet befindet sich in der Flyschzone. Sandstein und Mergel sind die dominierenden Gesteine in der Region (Egger, 1989). Flächenhaft verbreitete Bodentypen sind Braunerde, Pseudogley und Gley (BFW, 2016). Das subozeanische Klima, die lange Vegetationsperiode und das Relief begünstigen eine intensive Grünlandbewirtschaftung. Das Ertragspotenzial ist im Untersuchungsgebiet relativ hoch. Auf frischen bis mäßig feuchten Standorten sind Trockenmasse-Erträge über $100 \mathrm{dt}$ pro Hektar und Jahr sowie jährlich sechs Grünlandnutzungen möglich. Die Weideperiode dauert je nach Witterung von Anfang/Mitte April bis Ende Oktober/Mitte November. Der Viehbesatz beträgt im Flachgau 1,6 bis 2,0 Großvieheinheiten (GVE) ha ${ }^{-1}$ und ist somit deutlich höher als im Bundesland Salzburg mit durchschnittlich 1,4 GVE ha-1 (Amt der Salzburger Landesregierung, 2014).

Der Flachgau wurde als Untersuchungsgebiet ausgewählt, weil in diesem Bezirk eine intensive Grünlandwirtschaft betrieben wird.

\subsection{Untersuchungsflächen}

Diese Studie wurde auf mineralische Dauergrünlandböden beschränkt. Die Untersuchungsflächen wurden aufgrund von Geländebegehungen, Bodenkarten und Befragungen von Landwirten nach folgenden Kriterien ausgewählt:

1. Die ehemalige und derzeitige Bewirtschaftungsform (Wiese, Mähweide, Weide) und Nutzungsintensität (Anzahl der Schnitte und/oder Weidegänge pro Jahr) der Untersuchungsfläche muss bekannt sein.
2. Eine langjährig (mehr als 10 Jahre) gleichbleibende und regionaltypische Grünlandbewirtschaftung muss gewährleistet sein.

3. Der Boden- und Vegetationstyp muss repräsentativ für das Dauergrünland sein.

4. Der Boden muss eine natürliche Bodenbildung und Horizontfolge aufweisen (kein planierter Boden) und der Skelettgehalt (Grobanteil) darf $10 \%$ nicht übersteigen.

Die Untersuchungsflächen $(\mathrm{n}=22)$ wurden nach ihrer Bewirtschaftungsform gegliedert. Einen Überblick über die Nutzungsintensität und Befahrungshäufigkeit der einzelnen Bewirtschaftungsformen gibt Tabelle 1. Die Mähwiesen wurden nach ihrer Schnitthäufigkeit in extensiv genutzte Mähwiesen (ein bis zwei Schnitte pro Jahr) und intensiv genutzte Mähwiesen (drei bis sechs Schnitte pro Jahr) unterteilt. Sie wurden weder im Frühling noch im Herbst beweidet. Fünf- bis sechsschnittige Mähwiesen wurden im Zuge der Futtergewinnung, Düngung und Bestandespflege mehr als $30 \mathrm{Mal}$ pro Jahr mit Grünlandmaschinen befahren. Die Weiden (Koppelweiden) wurden tagsüber mit Milchkühen (Fleckvieh) bestoßen. Die Nutzungshäufigkeit betrug fünf bis sechs Weidegänge pro Jahr. Die Besatzdichte variierte zwischen 15 und 31 GVE ha- ${ }^{-1}$. Auf den Weideflächen erfolgte keine Mahd zum Zweck der Futtergewinnung. Die Weiden wurden jährlich mit dem Traktor befahren. Die wichtigsten Gründe hierfür waren Düngerausbringung, Durchführung von Reinigungsschnitten zwischen den Weidegängen, Wasserversorgung der Weidetiere und Reparaturarbeiten an Weidezäunen. Die Mähweiden wurden während der Vegetationsperiode sowohl beweidet als auch gemäht. In Abhängigkeit von der Schnitthäufigkeit (zwei bis drei Schnitte pro Jahr) erfolgten jährlich ein bis zwei Weidenutzungen.

Tabelle 1. Nutzungsintensität (Anzahl der Schnitte und/oder Weidegänge pro Jahr) und Befahrungshäufigkeit (durchschnittliche Anzahl an jährlichen Traktorüberfahrten)

Table 1. Intensity of use (annual number of cuts and/or pasturing) and average number of tractor passes each year

\begin{tabular}{lccc}
\hline Bewirtschaftungsform & Anzahl & Nutzungsintensität & Befahrungshäufigkeit \\
\hline Referenzfläche & 2 & $1-2$ & 0 \\
Extensiv genutzte Mähwiese & 5 & $1-2$ & $5-10$ \\
Intensiv genutzte Mähwiese & 6 & $3-6$ & meist $>30$ \\
Mähweide & 3 & $3-4$ & $10-30$ \\
Weide & 6 & $5-6$ & $5-10$ \\
\hline
\end{tabular}

Anzahl = Anzahl der Untersuchungsflächen 
Für die bodenphysikalische Beurteilung einer Bodenverdichtung sind Vergleichswerte von normalverdichteten Grünlandböden notwendig. Als Referenzflächen für den natürlichen Verdichtungszustand von Dauergrünlandböden im Untersuchungsgebiet dienten zwei Mähwiesen, die in den vergangenen 10 Jahren weder beweidet noch mit schweren Maschinen befahren wurden. Die Mahd erfolgte mittels Motormäher oder mit der Sense. Mit Ausnahme der Referenzflächen wurden alle Untersuchungsflächen regelmäßig mit Wirtschaftsdünger gedüngt.

Da physikalische Bodeneigenschaften auf engstem Raum stark schwanken, wurden die bodenkundlichen Untersuchungen nicht auf der gesamten Grünlandfläche, sondern nur auf einer homogenen und repräsentativen Teilfläche von $25 \times 25 \mathrm{~m}$ im Zeitraum Juli bis August 2016 durchgeführt. Die Seehöhe der Untersuchungsflächen betrug 520 bis $635 \mathrm{~m}$. Die Hangneigung variierte zwischen $2^{\circ}$ und $13^{\circ}$, wobei der Großteil der Untersuchungsflächen eine Neigung von $3^{\circ}$ bis $5^{\circ}$ aufwies. Die Exposition war überwiegend Ost und Südost. Die Bodentypen waren mittel- bis tiefgründige Braunerden, Pseudogleye und Gleye. Die Braunerden waren häufig krumen-pseudovergleyt. Der Vegetationsdeckungsgrad auf den Untersuchungsflächen variierte in Abhängigkeit von der Bewirtschaftungsform und Nutzungsintensität zwischen $70 \%$ und $95 \%$. Hauptbestandesbildner in den Mähwiesen waren Lolium $x$ boucheanum (Bastard-Raygras), Alopecurus pratensis (Wiesen-Fuchsschwanz) und Dactylis glomerata (WiesenKnaulgras). In den Weiden dominierten L. perenne (Englisches Raygras) und Trifolium repens (Weiß-Klee).

In einer Koppelweide wurde auch die räumliche Variabilität der LD und des EW bestimmt. Am Weideeingang war die jährliche Druckbelastung durch Viehtritt und Befahren am höchsten. Die Weidefläche im zentralen Bereich der Koppel wurde sowohl beweidet als auch mehrmals pro Jahr befahren. Die Steilfläche innerhalb der Weidekoppel wurde ausschließlich beweidet und nicht befahren. Die Auszäunungsfläche außerhalb der Weidekoppel wurde nicht beweidet und nur selten befahren. Die Hangneigung beim Weideeingang und auf der Weidefläche betrug $8^{\circ}$, auf der Steilfläche 13-18 und auf der Auszäunungsfläche $13^{\circ}$. Die Exposition war auf allen Flächen Südost.

\subsection{Bodenkundliche Untersuchungen}

Die LD wurde mit $100-\mathrm{cm}^{3}$-Stechzylindern in 5-cm-Tiefenstufen-Intervallen in sechsfacher Wiederholung bis zu einer Bodentiefe von $30 \mathrm{~cm}$ nach Hartge und Horn (2009) bestimmt. Diese Untersuchungstiefe wurde ausgewählt, weil in der Bodenschicht 0-30 cm die größten nutzungsbedingten Veränderungen der Bodendichte zu erwarten sind (Naeth et al., 1990). Der EW wurde mit einem Penetrologger (Eijkelkamp; Sondierkonus: $2 \mathrm{~cm}^{2}$ Basisfläche; Konuswinkel: $60^{\circ}$ ) bis zu einer Bodentiefe von $30 \mathrm{~cm}$ in zehnfacher Wiederholung gemessen. Die Ablesung der Messwerte erfolgte in Tiefenstufen von $5 \mathrm{~cm}$. Die Messungen wurden in einem Zeitraum von zwei Tagen bei ähnlichen Witterungsverhältnissen durchgeführt. Der volumetrische Bodenwassergehalt wurde in der Bodentiefe 0-5 cm in dreifacher Wiederholung mit einem BodenfeuchteMessgerät (Bodenfeuchtesensor: SM 150, Delta-T Devices Ltd) bestimmt. Das PV wurde aus der LD und Dichte der Festsubstanz $\left(2,65 \mathrm{~g} / \mathrm{cm}^{3}\right)$ berechnet. Zusätzlich wurden auch relevante bodenchemische Parameter ( $\mathrm{pH}-$ Wert, organischer Kohlenstoff, Gesamt-Stickstoff) und die Bodenart bestimmt. Dazu wurden auf jeder Untersuchungsfläche Bodenproben horizontweise bis in $30 \mathrm{~cm}$ Bodentiefe entnommen. Fünf Einzelproben pro Horizont wurden zu einer Mischprobe vereinigt. Die Bestimmung des $\mathrm{pH}-$ Wertes erfolgte in einer 0,01 $\mathrm{M} \mathrm{CaCl}_{2}$-Lösung (ÖNORM L 1083). Die Konzentration an organischem Kohlenstoff $\left(\mathrm{C}_{\text {org }}\right)$ und Gesamt-Stickstoff $\left(\mathrm{N}_{\mathrm{r}}\right)$ wurde durch trockene Verbrennung bei $900{ }^{\circ} \mathrm{C}$ (ÖNORM L 1080, 1095) mittels Elementaranalyse (VarioMAX CNS, Elementar Analysensysteme $\mathrm{GmbH}$ ) bestimmt. Nachdem die Böden karbonatfrei waren, entspricht der Gesamt-Kohlenstoff dem organischen Kohlenstoff. Die Korngrößenverteilung des Bodens wurde mittels Pipettmethode bestimmt (ÖNORM L 1061-2).

\subsection{Vegetationsaufnahmen}

Auf allen Untersuchungsflächen wurde im Zeitraum Juli bis August 2016 das Vorkommen oder Fehlen von Bodenverdichtungszeigern erfasst. $\mathrm{Zu}$ dieser ökologischen Artengruppe zählen im österreichischen Dauergrünland vor allem Agrostis stolonifera (Kriech-Straußgras), Bellis perennis (Gänseblümchen), Matricaria discoidea (Knopf-Kamille), Plantago major ssp. major (Breit-Wegerich), Poa annua (Einjähriges Rispengras), Poa supina (Läger-Rispengras), Polygonum aviculare (Gewöhnlicher Vogelknöterich), Ranunculus repens (Kriech-Hahnenfuß) und Scorzoneroides (Leontodon) autumnalis (Herbst-Schuppenleuenzahn). Diese Arten haben ihren Verbreitungsschwerpunkt in Tritt- und Flutrasen (Dierschke und Briemle, 2002). Auf einer Fläche von $5 \times 5$ $\mathrm{m}$ wurden ihre Deckungsgrade geschätzt. 


\subsection{Statistische Analysen}

Alle Parameter wurden mit dem Shapiro-Wilk-Test auf Normalverteilung geprüft. Die Überprüfung der Varianzhomogenität erfolgte durch den Levene-Test. Unterschiede in den Mittelwerten wurden mittels Kruskal-Wallis-Test geprüft. Anschließende Post-hoc-Tests wurden durch die Prozedur NPAR1WAY (SAS) mittels Wilcoxon-Rangsummentests durchgeführt. Es erfolgte eine Alpha-Adjustierung nach der Methode von Bonferroni-Holm. Zur Analyse von Korrelationen wurden Rangkorrelationen nach Spearman durchgeführt. Das Signifikanzniveau lag bei $5 \%(p=0,05)$. Die statistische Auswertung der Daten erfolgte mit der Statistiksoftware R (Commander Version 2.2-1) sowie SAS (Version 9.4).

\section{Ergebnisse}

\subsection{Bodenart und bodenchemische Kennwerte}

Die untersuchten Dauergrünlandböden wiesen in der Bodentiefe 0-30 cm im Durchschnitt eine mäßig saure bis stark saure Bodenreaktion auf. Die durchschnittliche Konzentration an $\mathrm{C}_{\text {org }}$ variierte im Oberboden (A-Horizont, $0-10 \mathrm{~cm})$ zwischen 30 und $52 \mathrm{~g} \mathrm{~kg}^{-1}$ und im Unterboden (AB-, P- oder Go-Horizont, 10-30 cm) zwischen 14 und $20 \mathrm{~g} \mathrm{~kg}^{-1}$. Das durchschnittliche C:N-Verhältnis schwankte im Oberboden zwischen 9,6 und 11,1 und im Unterbo- den zwischen 10,3 und 11,6 (Tabelle 2). Die Böden unter Weiden, Mähweiden und intensiv genutzten Mähwiesen wiesen im Durchschnitt höhere pH-Werte im Ober- und Unterboden sowie engere C:N-Verhältnisse im Oberboden als die Böden unter extensiv genutzten Mähwiesen und Referenzflächen auf. Diese Differenzen können mit der stärkeren Düngung (Zufuhr von mineralischen Kationenbasen und Stickstoff) und mit dem rascheren Stoffumsatz in den intensiver genutzten Grünlandböden erklärt werden. Die Bodenarten waren in allen untersuchten Böden sandiger Lehm oder Lehm. Der Tongehalt variierte zwischen $19 \%$ und $36 \%$.

\subsection{Bodenwassergehalt}

Tabelle 3 zeigt den mittleren volumetrischen Bodenwassergehalt und den Wassersättigungsgrad des Porenraumes zum Zeitpunkt der Messung des EW. Der Wassergehalt in 0-5 cm Bodentiefe betrug unter Weiden, Mähweiden und Mähwiesen im Durchschnitt 38 bis 39 Vol.-\%. Somit wurde der EW bei vergleichbarem Bodenfeuchtegrad (nahe Feldkapazität) gemessen. In den Referenzböden hingegen war der Wassergehalt mit durchschnittlich 19 Vol.-\% relativ niedrig (deutlich unter der Feldkapazität). Nachdem die Witterung während der EW-Messungen ähnlich war, dürften relief- und bodenbedingte höhere Verdunstungs- und Sickerwasserverluste auf den Referenzflächen verantwortlich für die niedrigere Bodenfeuchte im Vergleich zu den

Tabelle 2. Bodenchemische Kennwerte und Korngrößenverteilung des Bodens (Durchschnittswerte)

Table 2. Soil chemical properties and particle size distribution (mean values)

\begin{tabular}{|c|c|c|c|c|c|c|c|c|c|c|c|c|c|c|}
\hline \multirow[t]{2}{*}{$\begin{array}{l}\text { Bewirtschaftungs- } \\
\text { form }\end{array}$} & \multirow[t]{2}{*}{ Anzahl } & \multirow[t]{2}{*}{$\begin{array}{l}\text { Bodentiefe } \\
\quad(\mathrm{cm})\end{array}$} & \multicolumn{2}{|c|}{$\mathrm{pH}$} & \multicolumn{2}{|c|}{$\begin{array}{c}\mathrm{C}_{\text {org }} \\
\left(\mathrm{g} \mathrm{kg}^{-1}\right)\end{array}$} & \multicolumn{2}{|c|}{$C: N$} & \multicolumn{2}{|c|}{ Sand } & \multicolumn{2}{|c|}{$\begin{array}{l}\text { Schluff } \\
(\%)\end{array}$} & \multicolumn{2}{|c|}{ Ton } \\
\hline & & & $\mathrm{aM}$ & (SD) & $\mathrm{aM}$ & (SD) & $\mathrm{aM}$ & (SD) & $\mathrm{aM}$ & (SD) & $\mathrm{aM}$ & (SD) & $\mathrm{aM}$ & (SD) \\
\hline \multirow[t]{2}{*}{ RF } & 2 & $0-10$ & 4,7 & $(0,74)$ & 29,9 & $(1,6)$ & 11,1 & $(0,00)$ & 27,0 & $(3,7)$ & 46,1 & $(3,0)$ & 26,9 & $(0,7)$ \\
\hline & & $10-30$ & 4,7 & $(0,88)$ & 20,2 & $(4,0)$ & 11,2 & $(1,32)$ & 26,8 & $(5,7)$ & 45,9 & $(4,6)$ & 27,3 & $(1,2)$ \\
\hline \multirow[t]{2}{*}{ EM } & 5 & $0-10$ & 4,9 & $(0,30)$ & 49,7 & $(14,0)$ & 10,5 & $(1,00)$ & 29,4 & $(2,2)$ & 44,2 & $(2,0)$ & 26,4 & $(1,8)$ \\
\hline & & $10-30$ & 4,9 & $(0,24)$ & 16,5 & $(6,7)$ & 11,4 & $(1,25)$ & 23,6 & $(10,4)$ & 43,6 & $(2,8)$ & 32,9 & $(9,8)$ \\
\hline \multirow[t]{2}{*}{ IM } & 6 & $0-10$ & 5,0 & $(0,33)$ & 37,6 & $(9,7)$ & 10,1 & $(0,43)$ & 30,3 & $(4,1)$ & 45,6 & $(2,2)$ & 24,1 & $(4,2)$ \\
\hline & & $10-30$ & 5,3 & $(0,33)$ & 13,8 & $(1,9)$ & 11,6 & $(0,58)$ & 33,3 & $(3,7)$ & 45,4 & $(2,2)$ & 21,3 & $(2,1)$ \\
\hline \multirow[t]{2}{*}{ MW } & 3 & $0-10$ & 5,1 & $(0,43)$ & 51,6 & $(19,1)$ & 9,6 & $(0,15)$ & 28,4 & $(1,0)$ & 46,0 & $(1,8)$ & 25,6 & $(2,4)$ \\
\hline & & $10-30$ & 5,3 & $(0,45)$ & 16,1 & $(5,5)$ & 10,3 & $(0,99)$ & 31,5 & $(2,5)$ & 45,6 & $(2,0)$ & 22,9 & $(1,9)$ \\
\hline \multirow[t]{2}{*}{ W } & 6 & $0-10$ & 5,7 & $(0,47)$ & 44,1 & $(5,1)$ & 10,2 & $(0,46)$ & 30,1 & $(3,6)$ & 44,2 & $(2,3)$ & 25,7 & $(3,2)$ \\
\hline & & $10-30$ & 5,6 & $(0,33)$ & 13,7 & $(3,0)$ & 11,5 & $(0,75)$ & 33,3 & $(4,8)$ & 43,9 & $(2,5)$ & 22,8 & $(2,9)$ \\
\hline
\end{tabular}

RF: Referenzfläche, EM: extensiv genutzte Mähwiese, IM: intensiv genutzte Mähwiese, MW: Mähweide, W: Weide, Anzahl: Anzahl der Untersuchungsflächen, pH: $\mathrm{pH}$ in $\mathrm{CaCl}_{2}$, aM: arithmetischer Mittelwert, (SD): Standardabweichung 
Tabelle 3. Volumetrischer Bodenwassergehalt (Durchschnittswerte) und Wassersättigungsgrad des Porenraumes zum Zeitpunkt der Messung des EW in der Bodentiefe $0-5 \mathrm{~cm}$

Table 3. Volumetric soil water content (mean values) and degree of water saturation of the soil pore space at the time of penetration resistance measurements in the layer $0-5 \mathrm{~cm}$

\begin{tabular}{lcccc}
\hline $\begin{array}{l}\text { Bewirtschaf- } \\
\text { tungsform }\end{array}$ & Anzahl & \multicolumn{2}{c}{$\begin{array}{c}\text { Wassergehalt } \\
\text { (Vol.-\%) }\end{array}$} & $\begin{array}{c}\text { Wassersätti- } \\
\text { gungsgrad }\end{array}$ \\
\hline RF & aM & (SD) & \\
EM & 2 & 19 & $(4,83)$ & 0,28 \\
IM & 5 & 39 & $(5,64)$ & 0,56 \\
MW & 6 & 38 & $(4,54)$ & 0,58 \\
W & 3 & 39 & $(4,14)$ & 0,64 \\
\hline
\end{tabular}

RF: Referenzfläche, EM: extensiv genutzte Mähwiese, IM: intensiv genutzte Mähwiese, MW: Mähweide, W: Weide, Anzahl: Anzahl der Untersuchungsflächen, aM: arithmetischer Mittelwert, (SD): Standardabweichung

Weide-, Mähweide- und Wiesenflächen sein. Der Wassersättigungsgrad des Porenraumes war in den Referenzböden am niedrigsten und in den Weide- und Mähweideböden im Durchschnitt am höchsten. Zwischen dem volumetrischen Bodenwassergehalt und dem EW bzw. der LD konnte kein statistischer Zusammenhang festgestellt werden.

\subsection{Bodenphysikalische Kennwerte}

Tabelle 4 gibt Auskunft über die mittlere LD der Grünlandböden bis in $30 \mathrm{~cm}$ Tiefe. Der niedrigste Einzelwert an einem Standort $\left(0,51 \mathrm{~g} / \mathrm{cm}^{3}\right)$ wurde in einem extensiv genutzten Wiesenboden in $0-5 \mathrm{~cm}$ Tiefe und der Höchstwert $\left(1,69 \mathrm{~g} / \mathrm{cm}^{3}\right)$ in einem Weideboden in $20-25 \mathrm{~cm}$ Tiefe gemessen. Die LD war in den Referenzböden und extensiv genutzten Wiesenböden im Durchschnitt in allen Tiefenstufen niedriger als in den intensiv genutzten Wiesen-, Mähweide- und Weideböden. Die Messwerte variierten in den extensiv genutzten Grünlandböden zwischen $0,78 \mathrm{~g} / \mathrm{cm}^{3}$ in $0-5 \mathrm{~cm}$ Tiefe und $1,36 \mathrm{~g} / \mathrm{cm}^{3}$ in $25-30 \mathrm{~cm}$ Tiefe. In den intensiv genutzten Grünlandböden schwankte die LD von $0,90 \mathrm{bis} 1,55 \mathrm{~g} / \mathrm{cm}^{3}$. In Weideböden war die LD in der Tiefenstufe $0-15 \mathrm{~cm}$ und in Mähweideböden in 0-10 cm Tiefe signifikant höher als in Wiesenböden. Mähweide- und Weideböden zeigten in keiner Tiefenstufe signifikante Unterschiede hinsichtlich LD. Extensiv genutzte Wiesenböden verzeichneten in allen Tiefenstufen eine signifikant niedrigere LD als intensiv genutzte Wiesenböden. Der Unterschied zwischen den Referenzböden und extensiv genutzten Wiesenböden hinsichtlich LD war in allen Untersuchungstiefen gering und mit einer Ausnahme $(5-10 \mathrm{~cm})$ statistisch nicht signifikant. Die LD korrelierte höchst signifikant negativ mit der $\mathrm{C}_{\text {org }}$-Konzentration $\left(r^{2}=-0,82, p<0,001\right)$ und mit dem Tongehalt $\left(r^{2}=-0,55\right.$, $\mathrm{p}<0,001)$ im Boden. Eine signifikant positive Korrelation gab es mit dem Sandgehalt $\left(r^{2}=0,49, p<0,05\right)$. Der Schluffgehalt hingegen korrelierte nicht mit der LD.

In Tabelle 5 ist der mittlere EW der Grünlandböden bis in $30 \mathrm{~cm}$ Tiefe angeführt. Der EW wies vor allem im AHorizont auf den einzelnen Standorten eine hohe räumliche Variabilität auf, welche mit zunehmender Bodentiefe tendenziell abnahm. Der Vergleich der Variationskoeffizienten (V) zeigt, dass die Streuung der Einzelmesswerte in allen Tiefenstufen beim EW (V: 12-44 \%) deutlich größer als bei der LD (V: 3-16 \%) war. Für den EW sind daher zahlreiche Wiederholungsmessungen (mindestens 10 Einzelmessungen pro Standort) erforderlich. Der niedrigste Einzelwert für den EW an einem Standort $(0,73 \mathrm{MPa})$

Tabelle 4. Lagerungsdichte $\left(\mathrm{g} \mathrm{cm}^{-3}\right)$ für die einzelnen Bewirtschaftungsformen (Durchschnittswerte)

Table 4. Soil bulk density $\left(\mathrm{g} \mathrm{cm}^{-3}\right)$ of individual management systems (mean values)

\begin{tabular}{|c|c|c|c|c|c|c|c|c|c|c|}
\hline \multirow{2}{*}{$\begin{array}{l}\text { Bodentiefe } \\
{[\mathrm{cm}]}\end{array}$} & \multicolumn{2}{|c|}{$\mathrm{RF}$} & \multicolumn{2}{|c|}{ EM } & \multicolumn{2}{|c|}{ IM } & \multicolumn{2}{|c|}{ MW } & \multicolumn{2}{|c|}{ W } \\
\hline & $\mathrm{aM}$ & (SD) & $\mathrm{aM}$ & (SD) & $\mathrm{aM}$ & (SD) & $\mathrm{aM}$ & (SD) & $\mathrm{aM}$ & (SD) \\
\hline $0-5$ & 0,85 & $(0,07)$ & 0,78 & $(0,13)$ & 0,90 & $(0,12)$ & 1,01 & $(0,11)$ & 1,02 & $(0,12)$ \\
\hline $5-10$ & 0,95 & $(0,08)$ & 1,02 & $(0,09)$ & 1,10 & $(0,10)$ & 1,23 & $(0,14)$ & 1,29 & $(0,04)$ \\
\hline $10-15$ & 1,11 & $(0,06)$ & 1,13 & $(0,06)$ & 1,28 & $(0,13)$ & 1,37 & $(0,14)$ & 1,46 & $(0,08)$ \\
\hline $15-20$ & 1,24 & $(0,04)$ & 1,21 & $(0,08)$ & 1,42 & $(0,11)$ & 1,52 & $(0,09)$ & 1,44 & $(0,10)$ \\
\hline $20-25$ & 1,36 & $(0,04)$ & 1,33 & $(0,08)$ & 1,49 & $(0,06)$ & 1,55 & $(0,09)$ & 1,51 & $(0,10)$ \\
\hline $25-30$ & 1,34 & $(0,08)$ & 1,36 & $(0,11)$ & 1,55 & $(0,06)$ & 1,53 & $(0,07)$ & 1,54 & $(0,05)$ \\
\hline
\end{tabular}

RF: Referenzfläche ( $n=2)$, EM: extensiv genutzte Mähwiese ( $n=5)$, IM: intensiv genutzte Mähwiese ( $n=6)$, MW: Mähweide $(n=3)$, W: Weide ( $n=6)$, aM: arithmetischer Mittelwert, (SD): Standardabweichung 
wurde in einer extensiv genutzten Mähwiese in 2-5 cm Bodentiefe und der Höchstwert (2,36 MPa) in einer Weide in 10-15 cm Bodentiefe gemessen. In den Referenzböden und extensiv genutzten Wiesenböden nahm der EW innerhalb der Bodenschicht 0-30 cm kontinuierlich mit der Tiefe zu (von durchschnittlich 0,84 bzw. 0,86 auf 1,33 bzw. 1,21 MPa). In den Weideböden wurde im Durchschnitt der höchste EW in 10-15 cm Tiefe (2,17 MPa), in den Mähweideböden in 15-20 cm Tiefe (2,10 MPa) und in den intensiv genutzten Wiesenböden in $20-25 \mathrm{~cm}$ Tiefe (1,54 MPa) gemessen. Die Weide- und Mähweideböden wiesen bis in eine Tiefe von $30 \mathrm{~cm}$ signifikant höhere EW als die Wiesenböden auf. In den Mähweideböden war der EW in der Bodentiefe $2-15 \mathrm{~cm}$ signifikant niedriger als in den Weideböden. In der Tiefenstufe 15-20 cm konnte kein statistisch signifikanter Unterschied festgestellt werden. In der Bodentiefe 20-30 cm wiesen die Mähweideböden signifikant höhere Messwerte auf. Der EW der intensiv genutzten Wiesenböden lag in allen untersuchten Tiefenstufen signifikant über jenem der extensiv genutzten Wiesenböden. Der Unterschied zwischen den Referenzböden und extensiv genutzten Wiesenböden hinsichtlich EW war in allen Untersuchungstiefen gering und mit einer Ausnahme $(5-10 \mathrm{~cm})$ statistisch nicht signifikant. Der EW korrelierte höchst signifikant positiv mit der $\operatorname{LD}\left(r^{2}=0,60\right.$, $\mathrm{p}<0,001)$ und signifikant negativ mit der $\mathrm{C}_{\text {org }}$-Konzentration $\left(r^{2}=-0,32, p<0,05\right)$. Keine Korrelation gab es mit dem Sand-, Schluff- oder Tongehalt.

Das PV, berechnet aus der mittleren LD der einzelnen Bewirtschaftungsformen, variierte in der Bodenschicht 0-30 $\mathrm{cm}$ in Abhängigkeit von der Bodentiefe, Bewirtschaftungsform und Nutzungsintensität zwischen $41 \%$ und $70 \%$ (Abbildung 1). Im A-Horizont $(0-10 \mathrm{~cm})$ lagen die Werte bei allen Bewirtschaftungsformen über $50 \%$. In den Re- ferenzböden und extensiv genutzten Wiesenböden betrug das PV von der Bodenoberfläche bis in $20 \mathrm{~cm}$ Tiefe mehr als $50 \%$. Ein PV von unter $50 \%$ wurde in den Weideund Mähweideböden bereits ab $10 \mathrm{~cm}$ Tiefe festgestellt. Die Referenzböden und extensiv genutzten Wiesenböden hatten in allen Tiefenstufen höhere PV als die intensiv genutzten Wiesen-, Mähweide- und Weideböden.

In einer Koppelweide wurde die räumliche Variabilität der LD und des EW bestimmt. Die Ergebnisse der bodenphysikalischen Messungen sind in Tabelle 6 zusammengefasst. Beim Weideeingang wurde in allen Tiefenstufen die höchste LD und in der Auszäunungsfläche mit einer Ausnahme $(25-30 \mathrm{~cm})$ die niedrigste LD gemessen. Der Unterschied zwischen befahrener Weidefläche und unbefahrener Steilfläche hinsichtlich LD war in der Bodentiefe 5-25 cm gering. In 0-5 cm Bodentiefe war die LD in der Steilfläche und in 25-30 cm Bodentiefe in der Weidefläche vergleichsweise höher. Der EW nahm in der Auszäunungsfläche (Bodenwassergehalt: 30 Vol.-\%) innerhalb der Bodenschicht 0-30 cm mit der Bodentiefe kontinuierlich von $0,73 \mathrm{MPa}$ auf 1,26 MPa zu. Er war gegenüber den anderen Teilflächen in allen Tiefenstufen wesentlich niedriger. Mit Ausnahme der Bodenschicht $2-5 \mathrm{~cm}$ war der EW in der Weidefläche (Bodenwassergehalt: 32 Vol.-\%) höher als in der Steilfläche (Bodenwassergehalt: 27 Vol.$\%)$. Der Höchstwert wurde in der Weidefläche $(2,12 \mathrm{MPa})$ in 5-10 cm und in der Steilfläche $(2,10 \mathrm{MPa})$ in $2-5 \mathrm{~cm}$ Bodentiefe festgestellt. Aus den Messwerten (LD, EW) ist abzuleiten, dass die Verdichtungswirkung durch Viehtritt in steilen Hanglagen in der obersten Bodenschicht $(0-5 \mathrm{~cm})$ besonders groß ist. Verantwortlich dafür ist der steigende Kontaktflächendruck mit zunehmender Hangneigung. Beim Weideeingang war der Bodenwassergehalt in $0-5 \mathrm{~cm}$ Tiefe mit 52 Vol.-\% am höchsten. Der EW war

Tabelle 5. Eindringwiderstand (MPa) für die einzelnen Bewirtschaftungsformen (Durchschnittswerte)

Table 5. Penetration resistance (MPa) of individual management systems (mean values)

\begin{tabular}{|c|c|c|c|c|c|c|c|c|c|c|}
\hline \multirow{2}{*}{$\begin{array}{l}\text { Bodentiefe } \\
{[\mathrm{cm}]}\end{array}$} & \multicolumn{2}{|c|}{$\mathrm{RF}$} & \multicolumn{2}{|c|}{ EM } & \multicolumn{2}{|c|}{ IM } & \multicolumn{2}{|c|}{ MW } & \multicolumn{2}{|c|}{ W } \\
\hline & $\mathrm{aM}$ & (SD) & $\mathrm{aM}$ & (SD) & $\mathrm{aM}$ & (SD) & $\mathrm{aM}$ & (SD) & $\mathrm{aM}$ & (SD) \\
\hline $2-5$ & 0,84 & $(0,37)$ & 0,86 & $(0,35)$ & 0,98 & $(0,35)$ & 1,56 & $(0,42)$ & 1,88 & $(0,51)$ \\
\hline $5-10$ & 1,06 & $(0,31)$ & 1,00 & $(0,27)$ & 1,14 & $(0,34)$ & 1,82 & $(0,36)$ & 2,08 & $(0,39)$ \\
\hline $10-15$ & 1,09 & $(0,33)$ & 1,09 & $(0,23)$ & 1,34 & $(0,31)$ & 2,03 & $(0,35)$ & 2,17 & $(0,27)$ \\
\hline $15-20$ & 1,19 & $(0,30)$ & 1,18 & $(0,20)$ & 1,47 & $(0,26)$ & 2,10 & $(0,29)$ & 2,07 & $(0,25)$ \\
\hline $20-25$ & 1,26 & $(0,32)$ & 1,21 & $(0,22)$ & 1,54 & $(0,27)$ & 2,05 & $(0,31)$ & 1,95 & $(0,30)$ \\
\hline $25-30$ & 1,33 & $(0,35)$ & 1,21 & $(0,22)$ & 1,52 & $(0,29)$ & 1,97 & $(0,29)$ & 1,82 & $(0,35)$ \\
\hline
\end{tabular}

RF: Referenzfläche ( $n=2)$, EM: extensiv genutzte Mähwiese ( $n=5)$, IM: intensiv genutzte Mähwiese ( $n=6)$, MW: Mähweide $(n=3)$, W: Weide ( $n=6)$, aM: arithmetischer Mittelwert, (SD): Standardabweichung 


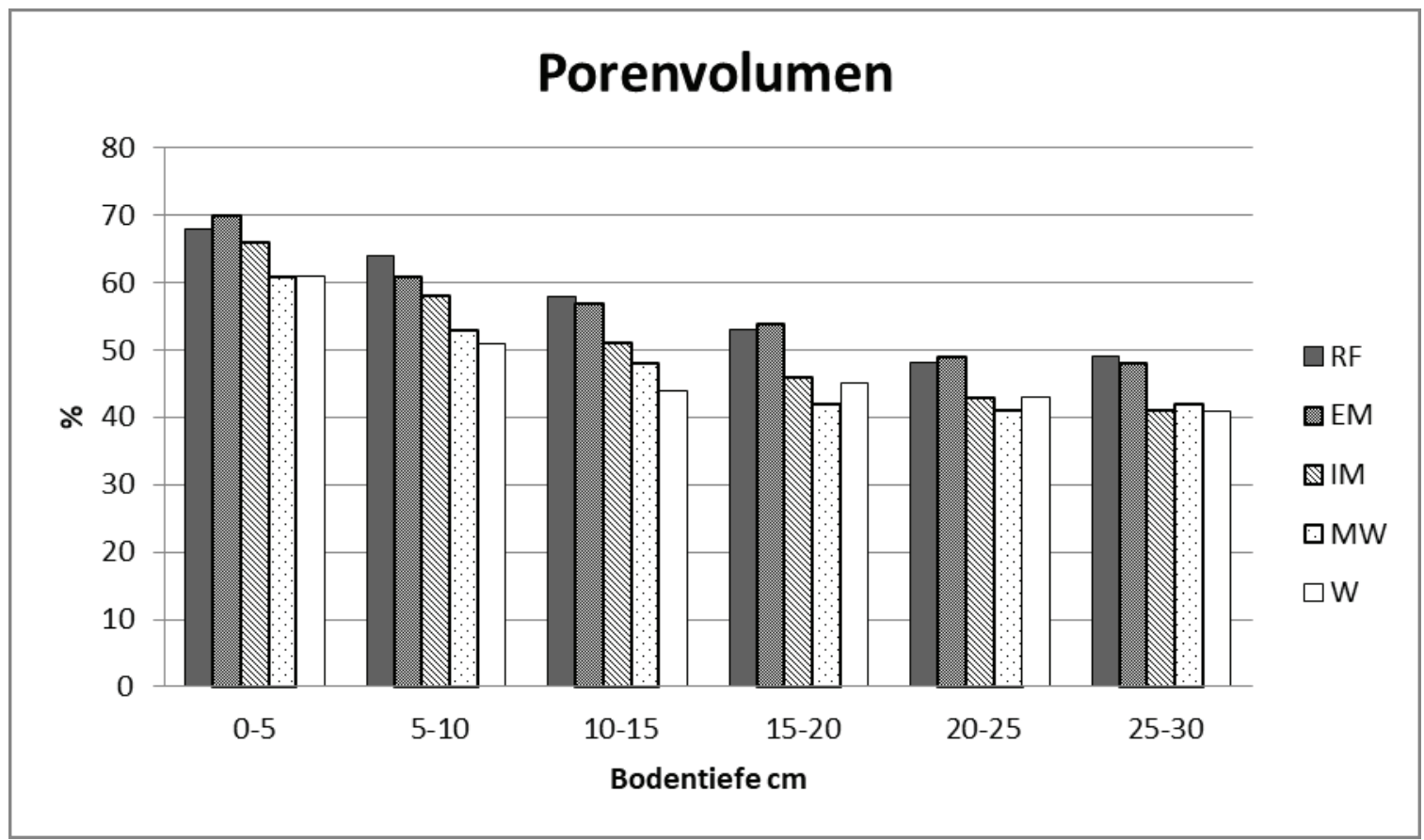

Abbildung 1. Porenvolumen für die einzelnen Bewirtschaftungsformen (Durchschnittswerte): RF: Referenzfläche ( $\mathrm{n}=2)$, EM: extensiv genutzte Mähwiese ( $n=5)$, IM: intensiv genutzte Mähwiese ( $n=6)$, MW: Mähweide ( $n=3)$, W: Weide ( $n=6)$. Porenvolumen, Annahme: Dichte der Festsubstanz: $2,65 \mathrm{~g} / \mathrm{cm}^{3}$

Figure 1. Pore volume of individual management systems (mean values): RF: reference area ( $\mathrm{n}=2)$, EM: extensivly used mowing meadow ( $\mathrm{n}=5)$, IM: intensivly used mowing meadow ( $\mathrm{n}=6)$, MW: mowing pasture $(\mathrm{n}=3)$, W: pasture $(\mathrm{n}=6)$. Pore volume, assumption: soil of solid substance: $2.65 \mathrm{~g} / \mathrm{cm}^{3}$

bis $10 \mathrm{~cm}$ Bodentiefe beträchtlich niedriger als in der Weidefläche und erst ab einer Tiefe von $15 \mathrm{~cm}$ deutlich höher. Der Höchstwert von 1,99 MPa wurde im Weideeingangsbereich in $10-15 \mathrm{~cm}$ Bodentiefe gemessen.

\subsection{Bodenverdichtungszeiger}

Tabelle 7 gibt Auskunft über die Häufigkeit des Vorkommens (Stetigkeit) und die Deckung der Bodenverdich- tungszeiger. Auf den Referenzflächen kamen keine Zeigerpflanzen für Bodenverdichtung vor. Auf den extensiv genutzten Mähwiesen wurde nur auf einem Standort $R$. repens mit geringer Individuenzahl beobachtet. Auf den intensiv genutzten Mähwiesen war der Deckungsgrad der Bodenverdichtungszeiger gering. Auf allen Untersuchungsflächen kam $R$. repens mit niedriger Deckung (2-3 \%) vor. Auf den Weiden und Mähweiden waren die Diversität und der Deckungsgrad der Bodenverdichtungszeiger deutlich

Tabelle 6. Lagerungsdichte $\left(\mathrm{g} \mathrm{cm}^{-3}\right)$ und Eindringwiderstand (MPa) innerhalb und außerhalb einer Weidekoppel (Durchschnittswerte) Table 6. Soil bulk density $\left(\mathrm{g} \mathrm{cm}^{-3}\right)$ and penetration resistance (MPa) within and outside of a paddock (mean values)

\begin{tabular}{|c|c|c|c|c|c|c|c|c|c|c|c|c|c|c|c|c|}
\hline \multirow{3}{*}{$\begin{array}{l}\text { Bodentiefe } \\
{[\mathrm{cm}]}\end{array}$} & \multicolumn{4}{|c|}{ Weideeingang } & \multicolumn{4}{|c|}{ Weidefläche } & \multicolumn{4}{|c|}{ Steilfläche } & \multicolumn{4}{|c|}{ Auszäunungsfläche } \\
\hline & \multicolumn{2}{|c|}{$\mathrm{LD}$} & \multicolumn{2}{|c|}{ EW } & \multicolumn{2}{|c|}{$\mathrm{LD}$} & \multicolumn{2}{|c|}{$\mathrm{EW}$} & \multicolumn{2}{|c|}{$\mathrm{LD}$} & \multicolumn{2}{|c|}{$\mathrm{EW}$} & \multicolumn{2}{|c|}{$\mathrm{LD}$} & \multicolumn{2}{|c|}{ EW } \\
\hline & $\mathrm{aM}$ & $(\mathrm{SD})$ & $\mathrm{aM}$ & $(\mathrm{SD})$ & $\mathrm{aM}$ & (SD) & $\mathrm{aM}$ & (SD) & $\mathrm{aM}$ & (SD) & $\mathrm{aM}$ & (SD) & $\mathrm{aM}$ & (SD) & $\mathrm{aM}$ & $(\mathrm{SD})$ \\
\hline $0 / 2-5^{*}$ & 1,20 & $(0,07)$ & 1,47 & $(0,65)$ & 1,06 & $(0,05)$ & 1,98 & $(0,59)$ & 1,12 & $(0,06)$ & 2,10 & $(0,60)$ & 0,83 & $(0,03)$ & 0,73 & $(0,36)$ \\
\hline $5-10$ & 1,36 & $(0,05)$ & 1,83 & $(0,23)$ & 1,30 & $(0,06)$ & 2,12 & $(0,32)$ & 1,28 & $(0,07)$ & 2,08 & $(0,62)$ & 1,02 & $(0,07)$ & 0,90 & $(0,27)$ \\
\hline $10-15$ & 1,52 & $(0,11)$ & 1,99 & $(0,22)$ & 1,40 & $(0,04)$ & 1,98 & $(0,21)$ & 1,41 & $(0,04)$ & 1,84 & $(0,40)$ & 1,16 & $(0,10)$ & 0,97 & $(0,22)$ \\
\hline $15-20$ & 1,54 & $(0,03)$ & 1,97 & $(0,33)$ & 1,40 & $(0,03)$ & 1,77 & $(0,17)$ & 1,41 & $(0,03)$ & 1,58 & $(0,32)$ & 1,38 & $(0.04)$ & 1,15 & $(0,14)$ \\
\hline $20-25$ & 1,60 & $(0,04)$ & 1,88 & $(0,25)$ & 1,47 & $(0,05)$ & 1,69 & $(0,16)$ & 1,48 & $(0,02)$ & 1,45 & $(0,22)$ & 1,34 & $(0,07)$ & 1,22 & $(0,13)$ \\
\hline $25-30$ & 1,64 & $(0,02)$ & 1,71 & $(0,19)$ & 1,58 & $(0,02)$ & 1,63 & $(0,18)$ & 1,40 & $(0,03)$ & 1,47 & $(0,19)$ & 1,44 & $(0,04)$ & 1,26 & $(0,12)$ \\
\hline
\end{tabular}

LD: Lagerungsdichte, EW: Eindringwiderstand, aM: arithmetischer Mittelwert, (SD): Standardabweichung, *Bodentiefe LD: 0-5 cm, EW: 2-5 cm 
höher als auf den Mähwiesen. Auf allen beweideten Standorten kam $R$. repens vor; der Deckungsgrad variierte von $3 \%$ bis $20 \%$. Auch Poa annua agg. (P. annua, P. supina) und $P$. major ssp. major waren immer präsent. $P$. annua agg. erreichte auf den beweideten Flächen einen Deckungsgrad von $10 \%$ bis $38 \%$. Im Eingangsbereich einer Koppelweide, wo der Tritteinfluss am größten ist, betrug die Gesamtdeckung der Bodenverdichtungszeiger $74 \%$; P. annua agg. und $R$. repens waren die dominierenden Pflanzenarten. Der Deckungsgrad der Bodenverdichtungszeiger korrelierte höchst signifikant positiv mit der mittleren $\mathrm{LD}\left(\mathrm{r}^{2}=0,78\right.$, $\mathrm{p}<0,001)$ und mit dem mittleren EW $\left(\mathrm{r}^{2}=0,88, \mathrm{p}<\right.$ $0,001)$ in $0-20 \mathrm{~cm}$ Bodentiefe.

\section{Diskussion}

Diese Studie wurde auf Flächen rinderhaltender Betriebe durchgeführt. Untersucht wurden typische österreichische Grünlandböden (Braunerde, Pseudogley, Gley) vorwiegend auf frischen oder wechselfeuchten Dauergrünlandstandorten. Die Bodenarten der Untersuchungsflächen waren sandiger Lehm oder Lehm. Die Bodenverdichtung wurde anhand der bodenphysikalischen Parameter LD, EW und PV sowie von Verdichtungszeigerpflanzen bestimmt. Die auf mineralischen Dauergrünlandböden erzielten Ergebnisse sind nicht übertragbar auf organische Dauergrünlandböden oder Böden unter Wechselgrünland.

\subsection{Bodenwassergehalt}

Der Wassersättigungsgrad des Porenraumes war in der Bodenschicht $0-5 \mathrm{~cm}$ vor allem in den intensiv genutzten
Weide- und Mähweideböden im Durchschnitt höher als in den Referenzböden und extensiv genutzten Wiesenböden. Insbesondere in überverdichteten Weide- und Mähweideböden sind deshalb die Pflanzenwurzeln vermutlich schlechter mit Sauerstoff versorgt als in normalverdichteten Grünlandböden. In den untersuchten Grünlandböden war der volumetrische Bodenwassergehalt mit wenigen Ausnahmen (Referenzflächen, Weideeingang) auf einem annähernd gleichen Niveau (nahe Feldkapazität). Daher waren Differenzen im Bodenwassergehalt nicht die wesentliche Ursache für die nutzungsbedingten Unterschiede im EW. Der EW wurde sehr wesentlich von der LD beeinflusst. Bei einer Bodenfeuchte nahe Feldkapazität nahm der EW mit steigender LD zu. Ähnliche Resultate wurden auch von anderen Autoren erzielt (Vepraskas, 1988; Martinez und Zinck, 2004). Allerdings wird mit steigendem Bodenwassergehalt der Zusammenhang zwischen EW und LD zunehmend schwächer (Vaz et al., 2001), weil der Boden plastischer wird.

\subsection{Bodenphysikalische Kennwerte}

Die Referenzböden repräsentieren normalverdichtete mineralische Dauergrünlandböden im Untersuchungsgebiet. In der Bodenschicht $0-20 \mathrm{~cm}$ betrug die LD weniger als $1,25 \mathrm{~g} / \mathrm{cm}^{3}$, der EW lag unter 1,20 MPa und das PV überstieg $50 \%$. Ähnliche Werte für normalverdichtete mineralische Dauergrünlandböden wurden auch in anderen Untersuchungsgebieten festgestellt (Bohner et al., 2006; Bohner und Tomanova, 2007). Abweichungen von diesen Referenzwerten weisen auf eine mögliche Überverdichtung des Bodens infolge intensiver Grünlandbewirtschaftung hin.

Tabelle 7. Häufigkeit des Vorkommens und Deckung der Bodenverdichtungszeiger

Table 7. Frequency of occurrence and cover of indicator species for soil compaction

\begin{tabular}{|c|c|c|c|c|c|c|c|c|c|c|c|c|c|c|c|c|c|}
\hline \multirow[t]{2}{*}{$\begin{array}{l}\text { Bewirtschafts- } \\
\text { ungsform }\end{array}$} & \multirow[t]{2}{*}{ Anzahl } & \multicolumn{2}{|c|}{$\begin{array}{l}\text { Agrostis } \\
\text { stolonifera }\end{array}$} & \multicolumn{2}{|c|}{$\begin{array}{c}\text { Bellis } \\
\text { perennis }\end{array}$} & \multicolumn{2}{|c|}{$\begin{array}{l}\text { Matricaria } \\
\text { discoidea }\end{array}$} & \multicolumn{2}{|c|}{$\begin{array}{l}\text { Plantago major } \\
\text { ssp. major }\end{array}$} & \multicolumn{2}{|c|}{$\begin{array}{c}\text { Poa } \\
\text { annua agg. }\end{array}$} & \multicolumn{2}{|c|}{$\begin{array}{l}\text { Polygonum } \\
\text { aviculare }\end{array}$} & \multicolumn{2}{|c|}{$\begin{array}{l}\text { Ranunculus } \\
\text { repens }\end{array}$} & \multicolumn{2}{|c|}{$\begin{array}{c}\text { Scorzoneroides } \\
\text { autumnalis }\end{array}$} \\
\hline & & $\mathrm{H}$ & $\mathrm{D}$ & $\mathrm{H}$ & $\mathrm{D}$ & $\mathrm{H}$ & $\mathrm{D}$ & $\mathrm{H}$ & D & $\mathrm{H}$ & D & $\mathrm{H}$ & $\mathrm{D}$ & $\mathrm{H}$ & D & $\mathrm{H}$ & $\mathrm{D}$ \\
\hline RF & 2 & 0 & 0 & 0 & 0 & 0 & 0 & 0 & 0 & 0 & 0 & 0 & 0 & 0 & 0 & 0 & 0 \\
\hline EM & 5 & 0 & 0 & 0 & 0 & 0 & 0 & 0 & 0 & 0 & 0 & 0 & 0 & 1 & 1 & 0 & 0 \\
\hline IM & 6 & 0 & 0 & 0 & 0 & 0 & 0 & 0 & 0 & 0 & 0 & 0 & 0 & 6 & $2-3$ & 1 & 1 \\
\hline MW & 3 & 1 & 3 & 2 & $2-3$ & 0 & 0 & 2 & 2 & 2 & $3-20$ & 1 & 1 & 3 & -10 & 2 & $1-2$ \\
\hline W & 6 & 4 & $2-10$ & 3 & $2-3$ & 2 & $1-2$ & 6 & $2-10$ & 6 & $10-38$ & 4 & $1-2$ & 6 & $3-20$ & 3 & 2 \\
\hline
\end{tabular}

RF: Referenzfläche, EM: extensiv genutzte Mähwiese, IM: intensiv genutzte Mähwiese, MW: Mähweide, W: Weide, Anzahl: Anzahl der Untersuchungsflächen, H: Häufigkeit des Vorkommens innerhalb der jeweiligen Pflanzenbestände, D: Deckungsgrad in \% 
Der Verdichtungsgrad von Dauergrünlandböden wurde maßgeblich von der Nutzungsintensität (jährliche Trittund Befahrungshäufigkeit) bestimmt. Die Bewirtschaftungsform und Hangneigung beeinflussten sehr wesentlich die Tiefe der Verdichtungszone im Grünlandboden. Die LD und der EW nahmen mit steigender Nutzungsintensität im Allgemeinen zu. In extensiv genutzten Mähwiesen (ein bis zwei Schnitte und weniger als 10 Überfahrten pro Jahr) war die LD nach der Klassifizierung von Schlichting et al. (1995) bis in $20 \mathrm{~cm}$ Bodentiefe in der Regel gering $\left(<1,25 \mathrm{~g} / \mathrm{cm}^{3}\right)$. Der EW lag unter 1,20 MPa. Im intensiv genutzten Dauergrünland hingegen war die LD in der Bodentiefe 5-20 cm häufig als mittel $\left(1,25-1,50 \mathrm{~g} / \mathrm{cm}^{3}\right)$ einzustufen und der EW überstieg an einigen Standorten 2,00 $\mathrm{MPa}$. In der Literatur wird eine $\mathrm{LD}$ von $1,30 \mathrm{~g} / \mathrm{cm}^{3}$ oder ein EW von 2,00 MPa häufig als Grenzwert genannt, bei dessen Überschreitung das Wurzelwachstum bei vielen Arten eingeschränkt ist (Materechera et al., 1991; Houlbrooke et al., 1997). Cook et al. (1996) haben bei Lolium perenne (Englisches Raygras), Agrostis capillaris (Rot-Straußgras) und Trifolium repens (Weiß-Klee) ein vermindertes Wurzel- und Sprosswachstum bei einem EW von 2,3 $\mathrm{MPa}$ festgestellt. In extensiv genutzten Mähwiesen gibt es demnach kein nutzungsbedingtes Bodenverdichtungsproblem. Bei langjährig intensiver Grünlandnutzung (mehr als drei Nutzungen pro Jahr) hingegen sind die Böden in der Regel mäßig überverdichtet; die Standortsverhältnisse und Konkurrenzbedingungen im Pflanzenbestand werden durch Bodenverdichtung modifiziert. Die stärkste Bodenverdichtung entsteht bei intensiver Beweidung mit Rindern. Durch Viehtritt wird der Boden vor allem in $5-15 \mathrm{~cm}$ Tiefe verdichtet. In steilen Hanglagen ist die Verdichtungswirkung im Oberboden besonders groß. Häufiges Befahren mit Grünlandmaschinen (mehr als 10 Überfahrten pro Jahr) führt zur Verdichtung zumindest bis $25 \mathrm{~cm}$ Bodentiefe. Im Untersuchungsgebiet sind intensiv genutzte Weideböden stärker verdichtet als intensiv genutzte Wiesenböden und die Verdichtungszone ist relativ stärker auf den Oberboden konzentriert. Der höhere Kontaktflächendruck durch Viehtritt (Moitzi und Boxberger, 2007) und die häufigere Druckbelastung bei intensiver Beweidung im Vergleich zum Befahren mit Grünlandmaschinen sind hierfür hauptverantwortlich. Die untersuchten Mähweiden nehmen hinsichtlich Bodenverdichtung (Ausmaß und Tiefe der Verdichtungszone) eine Mittelstellung zwischen Wiesen und Weiden ein. Diese Untersuchungsergebnisse stimmen mit jenen anderer Autoren überein. Nach Canillas und Salokhe (2001) wird die Bodenverdichtung maßgeb- lich von der jährlichen Befahrungshäufigkeit determiniert. Auch Hangneigung, Tritthäufigkeit und Tierart haben für die Bodenverdichtung eine große Bedeutung (Greenwood und McKenzie, 2001). Nach Kozlowski (1999) wird der Boden durch Tritt bevorzugt im Oberboden und durch Befahrung vor allem in mittlerer Tiefe verdichtet. Hassink und Neeteson (1991) fanden in Weideböden höhere LD als in Wiesenböden. Mulholland und Fullen (1991) haben auf intensiv genutzten Rinderweiden die stärkste Bodenverdichtung in 7-11 cm und Bohner und Tomanova (2007) in 5-10 cm Tiefe festgestellt. Einen erhöhten EW durch intensive Beweidung haben Naeth et al. (1990) vor allem in der Bodenschicht 2,5-10 cm gemessen. Eine langfristige Beweidung mit Rindern führt zu einer größeren LD und zu einem höheren EW im Oberboden (Evans et al., 2012). Auch eine langfristig intensive Beweidung mit Schafen und Ziegen kann eine Verdichtung im Oberboden verursachen (Zhou et al., 2010).

Die untersuchten Grünlandböden wiesen im A-Horizont meist ein großes PV (> 50 \%) auf. In den extensiv genutzten Mähwiesen betrug das PV innerhalb der Bodenschicht 0-20 cm im Durchschnitt mehr als $50 \%$. In den intensiv genutzten Grünlandböden traten innerhalb der Bodenschicht 0-20 cm häufig Verdichtungszonen mit PV zwischen $40 \%$ und $50 \%$ auf. Nach Sommer (1985) sind Böden locker gelagert, wenn das PV mehr als $50 \%$ ausmacht und sie sind stark verdichtet, wenn das PV weniger als $40 \%$ beträgt. Demnach sind die untersuchten extensiv genutzten Wiesenböden im Hauptwurzelraum locker gelagert, wohingegen die intensiv genutzten Dauergrünlandböden auf den meisten Standorten mäßig verdichtet sind. Eine Nutzungsintensivierung, vor allem eine intensive Beweidung, führt meist zu einer Abnahme des PV und zu einem Verlust an Grobporen im Oberboden (Herbin et al., 2011). Durch Viehtritt werden Makroporen vor allem in 5-10 cm Bodentiefe reduziert (Drewry, 2006). Dies hat negative Auswirkungen auf die Bodendurchlüftung und Wasserleitfähigkeit des Bodens (Drewry et al., 2008).

Die Bodenart hatte keinen bedeutenden Einfluss auf den EW in den untersuchten Grünlandböden. Die LD hingegen korrelierte höchst signifikant negativ mit dem Tongehalt und signifikant positiv mit dem Sandgehalt im Boden. Offensichtlich sind tonreiche (lehmige) Grünlandböden weniger verdichtungsempfindlich als sandreiche Grünlandböden. Tonreiche Böden haben insbesondere in niederschlagreichen Regionen durch regelmäßiges Quellen und Schrumpfen ein höheres natürliches Regenerationsvermögen als sandreiche Böden und sind daher bei inten- 
siver Grünlandbewirtschaftung weniger verdichtungsgefährdet. Tonreiche Böden sind allerdings aufgrund ihrer plastischen Eigenschaften im feuchten Zustand besonders empfindlich gegen Tritt- und Fahrschäden (Pietola et al., 2005; Bilotta et al., 2007). Unsere Untersuchungsergebnisse stimmen mit jenen anderer Autoren überein. Eine steigende LD mit zunehmendem Sandgehalt im Boden wurde auch von Newell-Price et al. (2013) festgestellt. Nach Canillas und Salokhe (2001) sinkt die Verdichtungsempfindlichkeit von Böden mit steigendem Tongehalt. Verdichtete Sandböden haben ein geringeres natürliches Regenerationsvermögen als verdichtete Tonböden (Nawaz et al., 2013). Durch Beweidung nimmt die LD in 0-5 cm Tiefe in sandigen Böden stärker zu als in lehmigen Böden (Hassink und Neeteson, 1991).

Die LD und der EW wiesen innerhalb einer Weidekoppel nutzungsbedingt eine hohe räumliche Variabilität auf. Insbesondere im Weideeingangsbereich war der Boden aufgrund der hohen Trittbelastung, beurteilt anhand der $\mathrm{LD}$, bis in $30 \mathrm{~cm}$ Tiefe überverdichtet und der volumetrische Bodenwassergehalt stark erhöht. Allerdings wurde die Verdichtung in 0-10 $\mathrm{cm}$ Bodentiefe durch den EW schlecht identifiziert. Vermutlich verhinderte die verdichtungsbedingte höhere Bodenfeuchte einen stärkeren Anstieg des EW in 0-10 cm Tiefe, weil der Boden plastischer wurde. Aus diesem Untersuchungsergebnis ist abzuleiten, dass zumindest während des Messzeitpunktes eine mechanische Behinderung des Wurzelwachstums im Oberboden unwahrscheinlich ist. Allerdings sollten wegen der feuchtebedingten zeitlichen Variabilität der Bodenfestigkeit EW-Messungen während der gesamten Vegetationsperiode durchgeführt werden. Unsere Untersuchungsergebnisse stimmen mit jenen anderer Autoren überein. Nach Kirkegaard et al. (1992) steigt der volumetrische Wassergehalt im Oberboden mit zunehmender LD, während die Wasserinfiltration und Bodenfestigkeit (gemessen als EW) sinken; der mechanische Stress für Pflanzenwurzeln wird dadurch vermindert. Eine erhöhte LD, ein vermindertes PV und ein erhöhter volumetrischer Bodenwassergehalt im Eingangsbereich einer Koppelweide wurde auch von Bohner und Tomanova (2007) festgestellt. Nach Hopkins und Patrick (1969) wird in verdichteten Lehmböden das Wurzelwachstum vor allem durch Sauerstoffmangel und selten aufgrund eines hohen EW eingeschränkt. Pietola et al. (2005) haben in der unmittelbaren Umgebung von Vieh-Tränken infolge hoher Trittbelastung eine geringe Wasserinfiltration und Festigkeit im tonreichen Oberboden sowie eine Verdichtung in tieferen Bodenschichten festgestellt.
In den untersuchten Grünlandböden nahmen mit steigender $\mathrm{C}_{\text {org }}-$ Konzentration die LD und der EW ab. Vor allem die $\mathrm{LD}$ war sehr stark von der $\mathrm{C}_{\text {org }}$-Konzentration im Boden abhängig. Ein hoher Humusgehalt im Oberboden verhindert eine starke Bodenverdichtung und erhöht somit die mechanische Belastbarkeit der Grünlandböden. Eine sinkende LD und abnehmende Verdichtungsempfindlichkeit von Böden mit steigendem Humusgehalt wurden auch in anderen Untersuchungen festgestellt (Soane, 1990; Heuscher et al., 2005; Herbin et al., 2011).

Die LD war in der humusreichen und stark durchwurzelten obersten Bodenschicht $(0-5 \mathrm{~cm})$ meist sehr gering (< $1,00 \mathrm{~g} / \mathrm{cm}^{3}$ in extensiv genutzten Wiesenböden) bis gering $\left(<1,25 \mathrm{~g} / \mathrm{cm}^{3}\right.$ in intensiv genutzten Grünlandböden). Das PV überstieg häufig $60 \%$. Der EW betrug im Durchschnitt in Wiesenböden weniger als 1,00 MPa und erreichte in Mähweide- und Weideböden Durchschnittswerte von 1,55 und 1,90 MPa. Somit dürfte in der Bodenschicht 0-5 $\mathrm{cm}$ weder ein hoher EW noch ein Sauerstoffmangel das Wurzelwachstum hemmen. Deswegen bilden die Pflanzen ihre Wurzelsysteme in überverdichteten Dauergrünlandböden bevorzugt in dieser obersten Bodenschicht (Bohner et al., 2016). Dieses Untersuchungsergebnis bestätigt jenes von Stahl et al. (2009), wonach Grünlandböden in 0-5 cm Tiefe locker gelagert sind. Der hohe Humusgehalt und die große Wurzelmasse sind dafür hauptverantwortlich.

\subsection{Bodenverdichtungszeiger}

Für das Vorkommen von Bodenverdichtungszeigern im Pflanzenbestand waren die Nutzungsintensität und der daraus resultierende Verdichtungsgrad des Grünlandbodens entscheidend. Bei hoher Nutzungsintensität (mehr als drei Nutzungen pro Jahr) können sich auf überverdichteten Grünlandböden Bodenverdichtungszeiger ansiedeln und stark ausbreiten. Die Bodenverdichtungszeiger haben einen hohen Lichtbedarf und profitieren deshalb von den besseren Lichtverhältnissen in Bodennähe bei häufiger Nutzung. Sie tolerieren verdichtete, staunasse, zeitweilig sauerstoffarme oder sauerstofffreie Böden, weil sie ein Durchlüftungsgewebe (Aerenchym) in den Wurzeln besitzen ( $R$. repens, $P$. major ssp. major) und/oder weil sie ihre Wurzeln in der besser durchlüfteten obersten Bodenschicht ausbreiten (P. annua, B. perennis) (Kutschera und Lichtenegger, 1982; 1992). Die Bodenverdichtungszeiger werden durch Reifendruck und Viehtritt mechanisch weniger stark geschädigt als viele typische Grünlandpflanzen. Außerdem profitieren sie von veränderten Konkurrenzbe- 
dingungen und von Lücken in der Grasnarbe resultierend aus dem Absterben stress- und störungsempfindlicher Arten. Deshalb sind Bodenverdichtungszeiger im Dauergrünland typische „Störzeiger“. Zwischen dem Deckungsgrad der Bodenverdichtungszeiger und den bodenphysikalischen Messwerten wurde eine höchst signifikant positive Korrelation festgestellt. Bodenverdichtungszeiger können daher zur Identifikation einer nutzungsbedingten Bodenverdichtung herangezogen werden. Ihr Deckungsgrad im Pflanzenbestand sollte alljährlich beobachtet werden, insbesondere wenn die Bewirtschaftungsform oder Nutzungsintensität verändert wurde. Auf den Referenzflächen und extensiv genutzten Mähwiesen fehlten Bodenverdichtungszeiger weitgehend. Die LD und der EW waren auf diesen Flächen bis in $20 \mathrm{~cm}$ Bodentiefe gering $(<1,25 \mathrm{~g} /$ $\mathrm{cm}^{3}$ bzw. $<1,20 \mathrm{MPa}$ ). In den intensiv genutzten Mähwiesen erreichten die Bodenverdichtungszeiger einen niedrigen Deckungsgrad, obwohl die Böden überverdichtet waren. Ursache hierfür war eine regelmäßige Nach- bzw. Übersaat, wodurch die Ansiedlung und Ausbreitung von Bodenverdichtungszeigern weitgehend verhindert wurde. Das Vorkommen von Bodenverdichtungszeigern im Pflanzenbestand ist somit für die Beurteilung einer Bodenverdichtung wichtiger als ihr Fehlen. Auf den intensiv beweideten Grünlandflächen erreichten die Bodenverdichtungszeiger ihre höchsten Deckungsgrade. In der Bodentiefe 5-20 $\mathrm{cm}$ waren die LD $\left(1,29-1,44 \mathrm{~g} / \mathrm{cm}^{3}\right)$ und der EW (2,07-2,17 MPa) signifikant höher als in den Referenzflächen und extensiv genutzten Mähwiesen. Vor allem $P$. annua agg., A. stolonifera, P. major ssp. major und $R$. repens profitierten von einer intensiven Trittbelastung und starken Bodenverdichtung. Sie zeigen bei Massenvorkommen (> $25 \%$ Gesamtdeckung) einen übernutzten Pflanzenstandort und eine Überschreitung der Intensivierungsgrenze an. Insbesondere $P$. annua neigt bei zu hoher Trittbelastung zur Dominanzbildung; sie ist ein relevanter Bioindikator für Überbeweidung. Auf den Untersuchungsflächen erreichte $R$. repens die höchste Stetigkeit unter den Bodenverdichtungszeigern. Der Kriech-Hahnenfuß ist im Gegensatz zu anderen Hahnenfuß-Arten $(R$. acris, $R$. bulbosus) keine Giftpflanze (Schubiger und Sachse, 1992). Er hat seinen Verbreitungsschwerpunkt in Feuchtwiesen und Flutrasen. $R$. repens kann auch auf überverdichteten, insbesondere krumen-pseudovergleyten, Grünlandböden einen hohen Deckungsgrad erreichen. Auf diesen Standorten hat er seinen zweiten Verbreitungsschwerpunkt. Seine Wurzeln haben einen ausgeprägten Fäulnisschutz (M. Sobotik, mündliche Mitteilung), daher toleriert er lan- ganhaltende Staunässe im Wurzelraum. Mit seinen oberirdischen Kriechtrieben kann er Lücken in der Grasnarbe rasch besiedeln. $R$. repens ist somit im Dauergrünland ein sensitiver Bioindikator für Bodenverdichtung, Staunässe, hohe Nutzungsintensität und Vegetationslücken. Die Bodenverdichtungszeiger sind überwiegend niedrigwüchsige, bodenblattreiche Kriech- und Rosettenpflanzen. Sie sind dadurch resistent gegenüber häufiger Störung durch Tritt, Mahd oder Verbiss. Wenn sich Bodenverdichtungszeiger zu Lasten von ertragreichen Futtergräsern im Pflanzenbestand infolge Bodenverdichtung stark ausbreiten, sinkt der Ertrag und der Grünlandboden ist schadverdichtet. Die Gefahr einer ertragsmindernden Schadverdichtung steigt in Lehmböden beträchtlich, wenn innerhalb der Bodenschicht 0-20 cm durch Viehtritt oder Befahren eine Verdichtungszone mit einer LD über $1,40 \mathrm{~g} / \mathrm{cm}^{3}$ oder einem EW größer als 2,00 MPa entsteht. Je mächtiger und grobporenärmer diese Verdichtungszone ist, umso ungünstiger sind die Auswirkungen einer Bodenschadverdichtung auf die Grünlandvegetation, den Ertrag und die Bewirtschaftbarkeit der Grünlandfläche.

Für das Vorkommen von Bodenverdichtungszeigern auf intensiv genutzten lehmigen Grünlandböden ist weder eine häufige Störung durch Mahd oder Beweidung noch ein hoher EW des Bodens für Pflanzenwurzeln (mechanischer Stress) entscheidend. Die verdichtungsbedingte periodische Staunässe im Oberboden und die schlechte Versorgung der Pflanzenwurzeln mit Sauerstoff während der Wachstumsperiode (Staunässe- und Sauerstoffmangelstress in der verdichteten Bodenschicht) dürften primär dafür verantwortlich sein. Feldbodenkundliche Befunde (deutliche nutzungsbedingte Staunässemerkmale in überverdichteten Grünlandböden insbesondere in niederschlagreichen Regionen, keine Bodenverdichtungszeiger auf locker gelagerten Böden unter Weidezäunen trotz ständigem Verbiss der Pflanzen), die Ergebnisse der bodenphysikalischen Messungen (höherer Wassersättigungsgrad des Porenraumes und somit schlechtere Sauerstoffverfügbarkeit für Pflanzenwurzeln in überverdichteten Böden im Vergleich zu normalverdichteten Böden, geringer EW bei hoher Bodenfeuchte in verdichteten Böden, EW meist < 2,00 MPa) und Literaturhinweise (Douglas und Crawford, 1993; Kozlowski, 1999; Glab, 2014) rechtfertigen diese Hypothese. Eine nutzungsbedingte Bodenverdichtung ist daher besonders ungünstig in kühlen, niederschlagreichen Regionen oder in niederschlagreichen Jahren, weil Ausmaß, Zeitdauer und Häufigkeit der Staunässe primär von den Klimaelementen Niederschlag und Lufttemperatur abhängen. 


\section{Schlussfolgerungen}

1. In Grünlandböden wird die LD sehr stark von der Bewirtschaftungsform und Nutzungsintensität (Tritt- und Befahrungshäufigkeit) beeinflusst. Bei der Berechnung von Kohlenstoff-, Nährstoff- oder Schadstoff-Vorräten darf deshalb für verschiedene Grünlandökosysteme keine einheitliche LD unterstellt werden.

2. In extensiv genutzten Mähwiesen war die LD bis in $20 \mathrm{~cm}$ Bodentiefe in der Regel gering $(<1,25 \mathrm{~g} /$ $\mathrm{cm}^{3}$ ) und der EW lag unter 1,20 MPa. Im intensiv genutzten Dauergrünland war die LD in der Bodentiefe 5-20 cm häufig als mittel $\left(1,25-1,50 \mathrm{~g} / \mathrm{cm}^{3}\right)$ einzustufen und der EW überstieg an einigen Standorten 2,00 MPa. Die stärkste Bodenverdichtung entsteht bei intensiver Beweidung mit Rindern. Durch Viehtritt wird der Boden insbesondere bis in $15 \mathrm{~cm}$ Tiefe verdichtet. Häufiges Befahren mit Grünlandmaschinen (mehr als 10 Überfahrten pro Jahr) bewirkt eine Verdichtung zumindest bis $25 \mathrm{~cm}$ Bodentiefe. Die Mähweiden nehmen hinsichtlich Bodenverdichtung (Ausmaß und Tiefe der Verdichtungszone) eine Mittelstellung zwischen Wiesen und Weiden ein.

3. Eine Bodenverdichtung kann die Artenzusammensetzung der Grünlandvegetation und das Ertragspotential des Grünlandstandortes insbesondere in kühlen, niederschlagreichen Regionen vor allem durch Staunässe und Sauerstoffmangel im Oberboden negativ beeinflussen.

4. Bodenverdichtungszeiger eignen sich zur flächenhaften Beurteilung des Verdichtungszustandes von Dauergrünlandböden. Ihr Vorkommen im Pflanzenbestand koinzidiert sehr gut mit der LD und dem EW in der Bodenschicht $0-20 \mathrm{~cm}$. R. repens (Kriech-Hahnenfuß) ist ein besonders sensitiver Bioindikator für Bodenverdichtung, Staunässe, hohe Nutzungsintensität und Vegetationslücken. Er eignet sich zur Früherkennung einer Bodenschadverdichtung.

5. Die Gefahr einer ertragsmindernden Schadverdichtung steigt in Lehmböden beträchtlich, wenn innerhalb der Bodenschicht 0-20 cm durch Viehtritt oder Befahren eine Verdichtungszone mit einer LD über $1,40 \mathrm{~g} / \mathrm{cm}^{3}$ oder einem EW größer als 2,00 MPa entsteht. Sobald diese vegetationsökologisch begründeten Schwellenwerte überschritten werden, ist eine Bodenverdichtung im Dauergrünland nicht mehr tolerierbar.

6. Bei der Bewertung der Daten aus der vorliegenden Untersuchung muss beachtet werden, dass sie in einem räumlich eng begrenzten Gebiet erhoben wurden. Das Datenmaterial ist noch zu gering, um allgemeingültige Aussagen über den Verdichtungszustand von österreichischen Dauergrünlandböden machen zu können. Für die Festlegung von bodenartspezifischen und feuchteabhängigen Schwellenwerten bezüglich Bodenschadverdichtung müssen zahlreiche weitere Daten auf verschiedenen Standorten in unterschiedlichen $\mathrm{Na}$ turräumen erhoben werden. Außerdem sollte geprüft werden, ob in schadverdichteten Dauergrünlandböden eine natürliche Regeneration der vegetationsökologisch relevanten bodenphysikalischen Parameter stattfindet und welcher Zeitbedarf hierfür erforderlich ist.

\section{Danksagung}

Wir danken Monika Kumpan, Josef Kaufmann und Lisa Egger für die Durchführung der Bodenanalysen.

\section{Literatur}

Amt der Salzburger Landesregierung (2014): Grüner Bericht - Bericht über die wirtschaftliche und soziale Lage der Salzburger Land- und Forstwirtschaft in den Jahren 2010 bis 2012. $128 \mathrm{~S}$.

BFW (Bundesforschungszentrum für Wald) (2016): Digitale Bodenkarte Österreichs. http://www.gis.lebensministerium.at/ebod. Abgerufen am 29. Dezember 2016.

Bengough, A.G., Bransby, M.F., Hans, J., McKenna, S.J., Roberts, T.J. und T.A. Valentine (2006): Root responses to soil physical conditions; growth dynamics from field to cell. Journal of Experimental Botany 57, 437-447.

Bilotta, G.S., Brazier, R.E. und P.M. Haygarth (2007): The impacts of grazing animals on the quality of soils, vegetation, and surface waters in intensively managed grasslands. Advances in Agronomy 94, 237-280.

BMLFUW (2016): Grüner Bericht 2016. Bericht über die Situation der österreichischen Land- und Forstwirtschaft, 57. Auflage, Wien, 263 S.

Bohner, A., Öhlinger, R. und O. Tomanova (2006): Auswirkungen der Grünlandbewirtschaftung und Flächenstilllegung auf Vegetation, Boden, mikrobielle Biomasse und Futterqualität. Die Bodenkultur 57, 33-45.

Bohner, A. und O. Tomanova (2007): Weideböden als Pflanzenstandorte. Mitteilungen der Österreichischen Bodenkundlichen Gesellschaft 74, 28-34. 
Bohner, A., Foldal, C.B. und R. Jandl (2016): Kohlenstoffspeicherung in Grünlandökosystemen - eine Fallstudie aus dem österreichischen Berggebiet. Die Bodenkultur 67, 225-237.

Breland, T.A. und S. Hansen (1996): Nitrogen mineralization and microbial biomass as affected by soil compaction. Soil Biology \& Biochemistry 28, 655-663.

Canillas, E.C. und V.M. Salokhe (2001): Regression analysis of some factors influencing soil compaction. Soil \& Tillage Research 61, 167-178.

Chyba, J., Kroulik, M., Kristof, K., Misiewicz, P.A. und K. Chaney (2014): Influence of soil compaction by farm machinery and livestock on water infiltration rate on grassland. Agronomy Research 12, 59-64.

Cook, A., Marriott, C.A., Seel, W. und C.E. Mullins (1996): Effects of soil mechanical impedance on root and shoot growth of Lolium perenne L., Agrostis capillaris and Trifolium repens $\mathrm{L}$. Journal of Experimental Botany 47, 1075-1084.

Dierschke, H. und G. Briemle (2002): Kulturgrasland. Ulmer Verlag, Stuttgart, 239 S.

Douglas, J.T. und C.E. Crawford (1993): The response of a ryegrass sward to wheel traffic and applied nitrogen. Grass and Forage Science 48, 91-100.

Drewry, J.J. (2006): Natural recovery of soil physical properties from treading damage of pastoral soils in New Zealand and Australia: A review. Agriculture, Ecosystems and Environment 114, 159-169.

Drewry, J.J., Cameron, K.C. und G.D. Buchan (2008): Pasture yield and soil physical property responses to soil compaction from treading and grazing - a review. Australian Journal of Soil Research 46, 237-256.

Egger, H. (1989): Zur Geologie der Flyschzone im Bundesland Salzburg. Jahrbuch der geologischen Bundesanstalt 132/2, 375-395.

Evans, C.R.W., Krzic, M., Broersma, K. und D.J. Thompson (2012): Long-term grazing effects on grassland soil properties in southern British Columbia. Canadian Journal of Soil Science 92, 685-693.

Glab, T. (2014): Effect of soil compaction and N fertilization on soil pore characteristics and physical quality of sandy loam soil under red clover/grass sward. Soil \& Tillage Research 144, 8-19.

Greacen, E.L. und R. Sands (1980): Compaction of forest soils. A review. Australian Journal of Soil Research 18, 163-189.

Greenwood, K.L. und B.M. McKenzie (2001): Grazing effects on soil physical properties and the consequences for pastures: a review. Australian Journal of Experimental Agriculture 41, 1231-1250.

Hakansson, I. und V.W. Medveder (1995): Protection of soils from mechanical overloading by establishing limits for stresses caused by heavy vehicles. Soil \& Tillage Research 35, 85-97.

Hansen, S. und L.R. Bakken (1993): $\mathrm{N}_{2} \mathrm{O}, \mathrm{CO}_{2}$ and $\mathrm{O}_{2}$ concentrations in soil air influenced by organic and inorganic fertilizers and soil compaction. Norwegian Journal of Agriculture Science 7, 1-10.

Hartge, K.H. und R. Horn (2009): Die physikalische Untersuchung von Böden. 4. Auflage, Schweizerbart, Stuttgart, 178 S.

Hartge, K.H. und R. Horn (2014): Einführung in die Bodenphysik. 4. Auflage, Schweizerbart, Stuttgart, 372 S.

Hassink, J. und J.J. Neeteson (1991): Effect of grassland management on the amounts of soil organic $\mathrm{N}$ and $\mathrm{C}$. Netherlands Journal of Agricultural Science 39, 225-236.

Herbin, T., Hennessy, D., Richards, K.G., Piwowarczyk, A., Murphy, J.J. und N.M. Holden (2011): The effects of dairy cow weight on selected soil physical properties indicative of compaction. Soil Use and Management 27, 36-44.

Herbauts, J., El Bayad, J. und W. Gruber (1996): Influence of logging traffic on the hydromorphic degradation of acid forest soils developed on loessic loam in middle Belgium. Forest Ecology and Management 87, 193-207.

Hernanz, J.L., Peixoto, H., Cerisola, C. und V. SánchezGirón (2000): An empirical model to predict soil bulk density profiles in field conditions using penetration resistance, moisture content and soil depth. Journal of Terramechanics 37, 167-184.

Heuscher, S.A., Brandt, C.C. und P.M. Jardine (2005): Using soil physical and chemical properties to estimate bulk density. Soil Science Society of America Journal 69, 51-56.

Hopkins, R.M. und W.H. Patrick (1969): Combined effect of oxygen content and soil compaction on root penetration. Soil Science 108, 408-413.

Horn, R. und K.H. Hartge (2001): Das Befahren von Ackerflächen als Eingriff in den Bodenwasserhaushalt. Wasser \& Boden 53/9, 13-19.

Houlbrooke, D.J., Thom, E.R., Chapman, R. und C.D.A. McLay (1997): A study of the effects of soil bulk density on root and shoot growth of different ryegrass lines. New Zealand Journal of Agricultural Research 40, 429-435. 
Iijima, M. und J. Kato (2007): Combined soil physical stress of soil drying, anaerobiosis and mechanical impedance to seedling root growth of four crop species. Plant Production Science 10, 451-459.

Kirkegaard, J.A., Troedson, R.J., So, H.B. und B.L. Kushwaha (1992): The effect of compaction on the growth of pigeonpea on clay soils. II. Mechanisms of crop response and seasonal effects on an oxisol in a humid coastal environment. Soil \& Tillage Research 24, 129-147.

Kozlowski, T.T. (1999): Soil compaction and growth of woody plants. Scandinavian Journal of Forest Research 14, 596-619.

Kriedner, A. (1989): Beobachtungen an einem nordbayerischen Vorkommen von Spiranthes spiralis (L.) Chevall. Berichte aus den Arbeitskreisen Heimische Orchideen 6, 79-81.

Kurz, I., O'Reilly, C.D. und H. Tunney (2006): Impact of cattle on soil physical properties and nutrient concentrations in overland flow from pasture in Ireland. Agriculture, Ecosystems and Environment 113, 378-390.

Kutschera, L. und E. Lichtenegger (1982): Wurzelatlas mitteleuropäischer Grünlandpflanzen. Band 1, Monocotyledoneae. Gustav Fischer Verlag, Stuttgart, 516 S.

Kutschera, L. und E. Lichtenegger (1992): Wurzelatlas mitteleuropäischer Grünlandpflanzen. Band 2, Pteridophyta und Dicotyledoneae. Gustav Fischer Verlag, Stuttgart, $851 \mathrm{~S}$.

Larink, O., Heisler, C., Söchtig, W., Lübben, B. und L. Wickenbrock (1994): Auswirkungen von Bodenverdichtungen auf die Meso- und Makrofauna. Landbauforschung Völkenrode SH 114, 129-145.

Lebert, M., Brunotte, J. und C. Sommer (2004): Ableitung von Kriterien zur Charakterisierung einer schädlichen Bodenveränderung, entstanden durch nutzungsbedingte Verdichtung von Böden/Regelungen zur Gefahrenabwehr. Texte Umweltbundesamt (Hrsg.) 46/04, Berlin, $122 \mathrm{~S}$.

Martinez, L.J. und J.A. Zinck (2004): Temporal variation of soil compaction and deterioration of soil quality in pasture areas of Colombian Amazonia. Soil \& Tillage Research 75, 3-17.

Materechera, S.A., Dexter, A.R. und A.M. Alston (1991): Penetration of very strong soils by seedling roots of different plant species. Plant and Soil 135, 31-41.

Meeuwig, R.O. (1965): Effects of seeding and grazing on infiltration capacity and soil stability of a subalpine range in central Utah. Journal of Range Management 18, 173-180.
Meredith, H.L. und W.H. Patrick, Jr. (1961): Effects of soil compaction on subsoil root penetration and physical properties of three soils in Louisiana. Agronomy Journal 53, 163-167.

Mohr, H.D. (1980): Einfluss der Bodeneigenschaften auf das Wurzelwachstum. Kali-Briefe 15, 305-316.

Moitzi, G. und J. Boxberger (2007): Vermeidung von Bodenschadverdichtungen beim Einsatz von schweren Landmaschinen - eine aktuelle Herausforderung. https://www.bmlfuw.gv.at/land/laendl_entwicklung/Online-Fachzeitschrift-Laendlicher-Raum/archiv/2007/ Moitzi.html. Abgerufen am 7. Juni 2017.

Mulholland, B. und M.A. Fullen (1991): Cattle trampling and soil compaction on loamy sands. Soil Use and Management 7, 189-193.

Murer, E., Sisák, I, Baumgarten, A. und P. Strauss (2006): Bewertung der Unterbodenverdichtung von Ackerböden im österreichischen Alpenvorland. Die Bodenkultur 63, 7-15.

Naeth, M.A., Pluth, D.J., Chanasyk, D.S., Bailey, A.W. und A.W. Fedkenheuer (1990): Soil compacting impacts of grazing in mixed prairie and fescue grassland ecosystems of Alberta. Canadian Journal of Soil Science 70, 157-167.

Nawaz, M.F., Bourrié, G. und F. Trolard (2013): Soil compaction impact and modelling. A review. Agronomy for Sustainable Development 33, 291-309.

Newell-Price, J.P., Whittingham, M.J., Chambers, B.J. und S. Peel (2013): Visual soil evaluation in relation to measured soil physical properties in a survey of grassland soil compaction in England and Wales. Soil \& Tillage Research 127, 65-73.

Peeters, A., Beaufoy, G., Canals, R.M., De Vliegher, A., Huyghe, C., Isselstein, J., Jones, G., Kessler, W., Kirilov, A., Mosquera-Losada, M.R., Nilsdotter-Linde, N., Parente, G., Peyraud, J.-L., Pickert, J., Plantureux, S., Porqueddu, C., Rataj, D., Stypinsky, P., Tonn, B., van den Pol- van Dasselaar, A., Vintu, V. und R.J. Wilkins (2014): Grassland term definitions and classifications adapted to the diversity of European grassland-based systems. In: Proceedings of the $25^{\text {th }}$ General Meeting of the European Grassland Federation. Grassland Science in Europe 19, 743-750.

Pietola, L., Horn, R. und M. Yli-Halla (2005): Effects of trampling by cattle on the hydraulic and mechanical properties of soil. Soil \& Tillage Research 82, 99-108.

Pulido, M., Schnabel, S., Lavado Contador, J.F., LozanoParra, J., Gómez-Gutiérrez, Á., Brevik, E.C. und A. Cerdà (2017): Reduction of the frequency of herbace- 
ous roots as an effect of soil compaction induced by heavy grazing in rangelands of SW Spain. Catena 158, 381-389.

Sitaula, B.K., Hansen, S., Sitaula, J.I.B. und L.R. Bakken (2000): Effects of soil compaction on $\mathrm{N}_{2} \mathrm{O}$ emission in agricultural soil. Chemosphere - Global Change Science 2, 367-371.

Schlichting, E., Blume, H.-P. und K. Stahr (1995): Bodenkundliches Praktikum. 2. Auflage, Blackwell Wissenschafts-Verlag, Berlin, 295 S.

Schubiger, F.X. und J. Sachse (1992): Bewertung des Kriechenden und des Scharfen Hahnenfußes als Futterpflanze. Landwirtschaft Schweiz 5, 589-592.

Soane, B.D. (1990): The role of organic matter in soil compactibility: a review of some practical aspects. Soil \& Tillage Research 16, 179-201.

Sommer, C. (1985): Ursachen und Folgen von Bodenverdichtungen sowie Möglichkeiten zu ihrer Verminderung. Landtechnik 40, 378-384.

Spiekers, H. und V. Potthast (2003): Erfolgreiche Milchviehfütterung. DLG Verlag, Frankfurt/Main, $448 \mathrm{~S}$.

Stahl, H., Marschall, K., Götze, H. und A. Freytag (2009): Bodendruck im Grünland. Schriftenreihe des Sächsi- schen Landesamtes für Umwelt, Landwirtschaft und Geologie, Heft 3/2009, 60 S.

Vaz, C.M.P., Bassoi, L.H. und J.W. Hopmans (2001): Contribution of water content and bulk density to field soil penetration resistance as measured by a combined cone penetrometer-TDR probe. Soil \& Tillage Research $60,35-42$.

Vepraskas, M.J. (1988): Bulk density values diagnostic of restricted root growth in coarse-textured soils. Soil Science Society of America Journal 52, 1117-1121.

Voorhees, W.B., Nelson, W.W. und G.W. Randall (1986): Extent and persistence of subsoil compaction caused by heavy axle loads. Soil Science Society of America Journal 50, 428-433.

ZAMG (Zentralanstalt für Meteorologie und Geodynamik) (2002): Klimadaten von Österreich 1971-2000. http://www.zamg.ac.at/fix/klima/oe71-00/klima2000/ klimadaten_oesterreich_1971_frame1.htm. Abgerufen am 7. Juni 2017.

Zhou, Z.C., Gan, Z.T., Shangguan, Z.P. und Z.B. Dong (2010): Effects of grazing on soil physical properties and soil erodibility in semiarid grassland of the Northern Loess Plateau (China). Catena 82, 87-91. 